Check for updates

Cite this: Soft Matter, 2019, 15, 1345

Received 13th August 2018, Accepted 23rd October 2018

DOI: $10.1039 / \mathrm{c} 8 \mathrm{sm} 01661 \mathrm{e}$

rsc.li/soft-matter-journal

\title{
Colloid supported lipid bilayers for self-assembly $\dagger$
}

\author{
Melissa Rinaldin, (D) $\ddagger^{\mathrm{ab}}$ Ruben W. Verweij, (D) $\ddagger^{\mathrm{a}}$ Indrani Chakraborty (D) ${ }^{\mathrm{c}}$ and \\ Daniela J. Kraft (DD $\star^{a}$
}

\begin{abstract}
The use of colloid supported lipid bilayers (CSLBs) has recently been extended to create colloidal joints, that enable the assembly of structures with internal degrees of flexibility, and to study lipid membranes on curved and closed geometries. These novel applications of CSLBs rely on previously unappreciated properties: the simultaneous fluidity of the bilayer, lateral mobility of inserted (linker) molecules and colloidal stability. Here we characterize every step in the manufacturing of CSLBs in view of these requirements using confocal microscopy and fluorescence recovery after photobleaching (FRAP). Specifically, we have studied the influence of different particle properties (roughness, surface charge, chemical composition, polymer coating) on the quality and mobility of the supported bilayer. We find that the insertion of lipopolymers in the bilayer can affect its homogeneity and fluidity. We improve the colloidal stability by inserting lipopolymers or double-stranded inert DNA into the bilayer. We include surface-mobile DNA linkers and use FRAP to characterize their lateral mobility both in their freely diffusive and bonded state. Finally, we demonstrate the self-assembly of flexibly linked structures from the CSLBs modified with surface-mobile DNA linkers. Our work offers a collection of experimental tools for working with CSLBs in applications ranging from controlled bottom-up self-assembly to model membrane studies.
\end{abstract}

\section{Introduction}

Colloid supported lipid bilayers (CSLBs) are used in a diverse range of research areas and applications, ${ }^{1}$ from drug delivery, ${ }^{2-4}$ bio-sensing, ${ }^{5,6}$ membrane manipulation ${ }^{7}$ and cell biology $^{8-10}$ to fundamental studies on lipid phase separation ${ }^{11,12}$ and self-assembly. ${ }^{13-16}$ The presence of a lipid bilayer around nano- or micrometer-sized solid particles or droplets provides biomimetic properties and a platform for further functionalization. One intriguing recent example used DNA-based linkers to functionalize the lipid bilayer, thereby enabling self-assembly of the underlying colloidal particles or droplets into flexible structures. ${ }^{13-18}$ Within such a structure, the colloidal elements

\footnotetext{
${ }^{a}$ Huygens-Kamerlingh Onnes Lab, Universiteit Leiden, P.O. Box 9504, 2300 RA Leiden, The Netherlands. E-mail: kraft@physics.leidenuniv.nl

${ }^{b}$ Instituut-Lorentz, Universiteit Leiden, P.O. Box 9506, 2300 RA Leiden, The Netherlands

${ }^{c}$ School of Chemistry, Raymond and Beverly Sackler Faculty of Exact Sciences, Tel Aviv University, Tel Aviv 69978, Israel

$\dagger$ Electronic supplementary information (ESI) available: The supporting information consists of 5 figures and 1 table: large fields of view of microscopy images; a table listing all employed DNA strands; a schematic representation of the particle tracking algorithm; a graph showing data from a previous paper re-analyzed using a different method; a microscopy picture of three CSLBs that feature membrane tubes; a microscopy picture of particles coated with DNA-stabilized SUVs. See DOI: 10.1039/c8sm01661e

\$ These authors contributed equally to the work.
}

can move over each other's surface while remaining strongly and specifically bonded. This new type of bonding enables fundamental studies on structures with internal degrees of flexibility, such as the self-assembly of novel crystal phases and their phase transitions. ${ }^{19-22}$ Furthermore, these complex colloids have great potential for smart drug delivery techniques, ${ }^{2}$ photonic band-gap materials ${ }^{23,24}$ and wet computing. ${ }^{25}$

CSLBs are particularly suitable and versatile building blocks for the assembly of floppy structures, because they combine the best qualities of free standing bilayers (vesicles) and colloids. Vesicles, upon application of linkers, ${ }^{26-29}$ can connect into flexible structures, but are unstable to small disturbances, heterogenous in size and easily deformable. Colloidal particles are available in diverse materials and with a variety of stable shapes, and can be assembled after functionalization with surface-bound DNA linkers. ${ }^{30-32}$ However, the obtained structures are often rigid due to the immobility of the linking groups on the particles' surface and are non-equilibrium structures due to a "hit-and-stick" aggregation process. ${ }^{33}$ Emulsions coated with lipid monolayers and DNA-linkers that are mobile on the droplet interface possess both interaction specificity and bond flexibility. ${ }^{17,18,34}$ Therefore, they assemble into flexible structures in a controlled fashion, but their shape is limited to spheres and they deform upon binding. Conversely, CSLBs consist of colloidal particles which provide a stable support for the lipid bilayer that is tunable in shape, size and material. 
The range of shapes for colloidal particles comprises, among others, spheres, cubes, rods, and (a)symmetric dumbbell particles, and their sizes range from hundreds of nanometers to several micrometers. They can be produced reliably with a narrow size distribution and are commercially available. Additionally, CSLBs feature a lipid bilayer on the surface of the colloids which creates a liquid film for molecules, such as DNA linkers, to freely move in. This allows for linking particles specifically, and yet flexibly, making the assembly of floppy structures possible. ${ }^{13-16,34}$

To obtain flexible instead of rigid structures, it is vital that the linker molecules which are inserted into the lipid bilayer are free to move over the surface of the CSLBs. Their lateral mobility relies on the fluidity and homogeneity of the bilayer, which in turn depend on the linker concentration ${ }^{16}$ and lipid composition. The lipids need to be in the fluid state under experimental conditions, and this may be impeded by bilayersurface interactions. Similarly, the success of experiments studying the phase separation of lipid bilayers on anisotropic colloidal supports relies on the fluidity and homogeneity of the bilayer. ${ }^{11,12}$ Finally, controlling the self-assembly pathway through complementary DNA linkers implies that all other nonspecific interactions need to be suppressed. In other words, CSLBs need to have sufficient colloidal stability. To the best of our knowledge, these requirements of membrane homogeneity and fluidity plus colloidal stability have not been studied simultaneously. However, they are of key importance for using CSLBs in self-assembly and model membrane studies, while possibly having wider implications for all other applications.

Here, we carefully characterize every stage in the preparation of CSLBs specifically related to these three properties. First, we study the effect of the material properties of the particles and the use of polymers on the membrane fluidity and homogeneity. Then, we investigate the influence of lipopolymers and inert double-stranded DNA on the colloidal stability of the CSLBs.

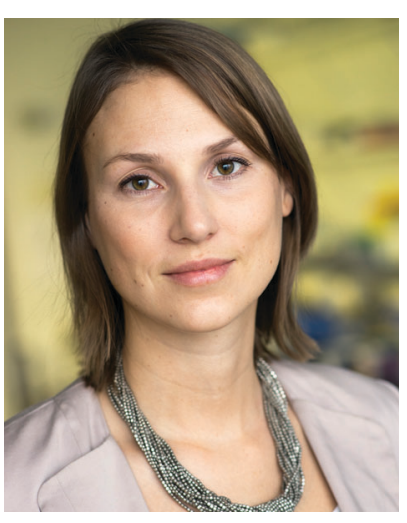

Daniela J. Kraft
Daniela Kraft is an Associate Professor in Soft Matter Physics at the Huygens-Kamerlingh Onnes Laboratory at Leiden University, The Netherlands. She obtained her PhD cum laude from the University of Utrecht, The Netherlands, under supervision of Willem Kegel. Supported by a Rubicon grant, she then joined the Center for Soft Matter Research at New York University, USA, as a postdoctoral researcher. In 2013, she moved to Leiden, where she established her own group. Her research focuses on self-assembly in biological and soft matter systems, ranging from anisotropic colloidal particles to lipid membranes, emulsions, and viruses. Dr Kraft has been awarded a VENI fellowship from the Netherlands Organisation for Scientific Research, an ERC starting grant and the paper of the year award 2017 from Biophysical Journal.
Subsequently, we include DNA-based linkers connected to hydrophobic anchors and characterize their diffusion in the bilayer. Finally, we show that when using the optimal experimental parameters determined by this study, CSLBs selfassemble into flexibly linked structures that are freely-jointed.

\section{Experimental section}

\section{Reagents}

Chemicals. 1-Palmitoyl-2-oleoyl-sn-glycero-3-phosphocholine (POPC), 1,2-dioleoyl-sn-glycero-3-phosphoethanolamine- $N$-(lissamine rhodamine B sulfonyl), 23-(dipyrrometheneboron difluoride)-24norcholesterol (TopFluor ${ }^{\mathbb{R}}$-Cholesterol), ( $\Delta$ 9-Cis) 1,2-dioleoylsn-glycero-3-phosphocholine (DOPC), 1,2-dioleoyl-sn-glycero-3phosphoethanolamine- $N$-[methoxy(polyethylene glycol)-2000] (DOPE-PEG(2000)), DOPE-PEG(3000) and DOPE-PEG(5000) were purchased from Avanti Polar Lipids. 4-(2-Hydroxyethyl)1-piperazineethanesulfonic acid (HEPES, $\geq 99.5 \%$ ) and calcium chloride $\left(\mathrm{CaCl}_{2}\right.$, calcium chloride dihydrate, $\left.\geq 99 \%\right)$ were purchased from Carl Roth. Sodium chloride ( $\mathrm{NaCl}$, extra pure), hydrogen peroxide $\left(\mathrm{H}_{2} \mathrm{O}_{2}, 35 \% \mathrm{w} / \mathrm{w}\right)$, acrylamide $(98.5 \%$, extra pure), $N, N, N^{\prime}, N^{\prime}$-tetramethylethylenediamine (TEMED, 99\%), ammonium persulfate (APS, 98\%), sodium hydroxide $(\mathrm{NaOH}$, 98.5\%) and sodium azide $\left(\mathrm{NaN}_{3}, 99 \%\right.$, extra pure) were purchased from Acros Organics. Hellmanex III, ammonium hydroxide $\left(\mathrm{NH}_{4} \mathrm{OH}, 28-30 \%\right.$ w/w), 3-(trimethoxysilyl)propyl methacrylate (TPM, 98\%), Pluronic F-127, dipotassium phosphate $\left(\mathrm{K}_{2} \mathrm{HPO}_{4}, \geq 99 \%\right)$, ethanol $(\geq 99.8 \%)$, sodium dodecyl sulfate (SDS, $\geq 98.5 \%$ ), polyvinylpyrrolidone (PVP, average $M_{\mathrm{w}}$ $40000)$, itaconic acid ( $\geq 99 \%)$, 3-aminopropyltriethoxysilane (99\%) and acetic acid (99.8\%) were purchased from SigmaAldrich. Magnesium chloride $\left(\mathrm{MgCl}_{2}\right.$, for analysis) was purchased from Merck. All solutions were prepared with Milli-Q water (Milli-Q Gradient A10).

Buffers. HEPES buffer type 1 was made with $115 \mathrm{mM} \mathrm{NaCl}$, $1.2 \mathrm{mM} \mathrm{CaCl}_{2}, 1.2 \mathrm{mM} \mathrm{MgCl}_{2}, 2.4 \mathrm{mM} \mathrm{K}_{2} \mathrm{HPO}_{4}$ and $20 \mathrm{mM}$ HEPES. HEPES buffer type 2 consisted of $10 \mathrm{mM}$ HEPES, $40 \mathrm{mM} \mathrm{NaCl}, 2 \mathrm{mM} \mathrm{CaCl}_{2}$ and $3 \mathrm{mM} \mathrm{NaN}_{3}$. HEPES buffer type 3 consisted of $10 \mathrm{mM}$ HEPES, $40 \mathrm{mM} \mathrm{NaCl}$ and $3 \mathrm{mM} \mathrm{NaN}_{3}$. The buffers were prepared by mixing all reagents in the appropriate amounts in fresh Milli-Q water. After mixing, the $\mathrm{pH}$ was adjusted to 7.4 using $\mathrm{NaOH}$.

Particles. Commercial silica spheres $((2.06 \pm 0.05) \mu \mathrm{m}$, $(2.12 \pm 0.06) \mu \mathrm{m},(7.0 \pm 0.29) \mu \mathrm{m})$ were synthesized by Microparticles $\mathrm{GmbH}$, using a Stöber method where tetraethoxysilane (TEOS) reacts with water and bases in an ethanolic solution (sol-gel process). Commercial polystyrene particles with sulfate groups $((2.0 \pm 0.3) \mu \mathrm{m})$ were obtained from Sigma Aldrich. Hematite cubic particles $((1.76 \pm 0.08) \mu \mathrm{m})$ were made following the protocol of Sugimoto et $\mathrm{al}^{35}$ and coated according to Rossi et al. ${ }^{36}$ Polystyrene3-(trimethoxysilyl)propyl methacrylate (polystyrene-TPM) particles $((1.24 \pm 0.04) \mu \mathrm{m})$ with varying asperity were synthesized and coated with silica following the protocol of Meester et al. ${ }^{37}$ TPM particles $((2.03 \pm 0.06) \mu \mathrm{m})$ were made following the protocol of Van der Wel et $a .^{38}$ TPM particles functionalized with carboxyl groups 
$((2.71 \pm 0.14) \mu \mathrm{m})$, or amino groups $((2.14 \pm 0.07) \mu \mathrm{m})$ were prepared by synthesizing according to ref. 38 and then functionalizing according to ref. 39. Briefly, amine or carboxylic acid groups were incorporated onto the TPM surface by addition of either 3-aminopropyltriethoxysilane or itaconic acid, respectively, during the emulsification stage. Polystyrene particles with carboxyl groups $((1.99 \pm 0.15) \mu \mathrm{m})$ were synthesized according to Appel et al. ${ }^{40}$ Polystyrene-TPM particles of spherical, symmetric and asymmetric dumbbell shape were made and coated with silica following the protocols reported in Rinaldin et al. ${ }^{11}$

DNA oligonucleotides. All DNA strands were synthesized as single-stranded DNA, purified using reverse phase highperformance liquid chromatography (HPLC-RP) and checked using matrix-assisted laser desorption/ionization time-of-flight mass spectrometry (Maldi-TOF MS) by Kaneka Eurogentec S.A. We used double-stranded inert DNA for steric stabilization and double-stranded DNA linkers with a sticky end for binding. Both types of DNA have a hydrophobic anchor (double stearyl/ double cholesterol for linker and double cholesterol for inert DNA) connected to a short carbon chain which is then connected to the oligonucleotide. The linkers are additionally functionalized with a fluorescent dye. All strands, including all functionalizations, are listed in Table S1 of the ESI. $\dagger$ These DNA strands were hybridized by mixing the single strands in a 1:1 molar ratio in HEPES buffer type 3 . The solution was then heated in an oven to $90{ }^{\circ} \mathrm{C}$ for $30 \mathrm{~min}$, after which the oven was turned off and the solution was allowed to cool down slowly overnight in the closed oven. Strand 1 and 2 are hybridized to form "10 nm Inert DNA", 3 and 4 are hybridized to form " $20 \mathrm{~nm}$ Inert DNA", 4 and 5 form " $20 \mathrm{~nm}$ Linker A", 4 and 6 make "20 nm Linker A", 7 and 8 form "30 nm Linker A" and, finally, 7 and 9 are hybridized to form "30 nm Linker $\mathrm{A}^{\prime \prime}$. . The linkers "A" have a single-stranded sticky end (indicated by cursive text in Table S1 of the ESI $\dagger$ ) that is complementary to the single-stranded end of linkers " $\mathrm{A}$ "."

\section{Preparation of CSLBs}

Typically, CSLBs were made by spontaneous spreading and fusion of small unilamellar vesicles (SUVs) on the particle surface. An SUV dispersion prepared via either extrusion or sonication was mixed with the particles, allowing a bilayer to spread on the surface for at least one hour. Subsequently the CSLBs were washed to remove excess SUVs. We observed no substantial differences in the obtained CSLBs between the two methods presented here.

CSLB preparation: method 1. $500 \mu \mathrm{g}$ of a mixture of DOPErhodamine (0.2 mole\%) and varying amounts of POPC and PEGylated lipids was dried by two hours of vacuum desiccation and then re-suspended to a $2 \mathrm{~g} \mathrm{~L}^{-1}$ dispersion with HEPES buffer type 1 . The solution was vortexed for $15 \mathrm{~min}$ to produce multilamellar vesicles. Then, the vesicle dispersion was extruded 21 times with a mini extruder (Avanti Polar Lipids) equipped with two $250 \mu \mathrm{L}$ gas-tight syringes (Hamilton), two drain discs and one nucleopore track-etch membrane (Whatman). The pore size of the membrane was 0.05 or $0.1 \mu \mathrm{m}$ for experiments with DOPE-PEG(2000) and DOPE-PEG(3000-5000), respectively.
The as-prepared $50 \mu \mathrm{L}$ of SUVs were added to $1 \mathrm{~mL}$ of $0.05 \% \mathrm{w} / \mathrm{v}$ of particles dispersed in HEPES buffer 1 . The particles were gently rotated for $1 \mathrm{~h}$. The resulting dispersion was centrifuged at 419 rcf for $1 \mathrm{~min}$ and the supernatant replaced with HEPES buffer type 1 to remove any SUVs present in the dispersion.

This method was used for all experiments regarding the influence of particle material, surface roughness and the effect of polymer insertion on the spreading and mobility of the lipid bilayer.

CSLB preparation: method 2. Typically, a lipid mixture of $98.9 \mathrm{~mol} \%$ DOPC, $1 \mathrm{~mol} \%$ DOPE-PEG(2000) and $0.1 \mathrm{~mol} \%$ DOPE-rhodamine or TopFluor-Cholesterol in chloroform was dried overnight in a glass vial covered with aluminum foil under vacuum desiccation. We investigated different PEGylated lipid lengths and molar ratios. After drying, $1 \mathrm{~mL}$ HEPES buffer type 2 or 3 was added to reach a concentration of $2 \mathrm{~g} \mathrm{~L}^{-1}$. The dispersion was vortexed for $30 \mathrm{~min}$, after which it became turbid. It was then transferred to a plastic test tube and ultrasonicated using a tip sonicator (Branson Sonifier SFX150) set to $30 \%$ of its maximum amplitude for a total time of $30 \mathrm{~min}$ using a pulsed sequence (18 s on/42 s off, total on time $9 \mathrm{~min}$ ) on ice to prevent heating. The SUV dispersion was then centrifuged for $45 \mathrm{~min}$ at 2029 rcf to sediment larger vesicles and titania particles originating from the tip. ${ }^{41} 200 \mu \mathrm{L}$ SUVs were taken from the top to isolate the smallest vesicles.

$17 \mu \mathrm{L}$ of $0.5 \mathrm{~g} \mathrm{~L}^{-1}$ SUVs in HEPES buffer 2 or 3 were mixed with $35 \mu \mathrm{L} 0.5 \% \mathrm{w} / \mathrm{v}$ of particles in Milli-Q water, leading to a surface ratio of SUVs: particles of $8: 1$. The dispersion was gently rotated for $1 \mathrm{~h}$. The particles were centrifuged at $43 \mathrm{rcf}$ for $2 \mathrm{~min}$ and the supernatant was replaced with HEPES buffer type 2 or 3 to remove any remaining free SUVs from the dispersion. Alternatively, the particles were allowed to sediment by gravity for $30 \mathrm{~min}$ instead of centrifuging and the supernatant was replaced.

This method was used for all experiments regarding the colloidal stability of CSLBs, the mobility of inserted DNA and the mobility of self-assembled CSLB clusters. For all experiments where DNA was inserted into the membrane of the CSLBs, buffer type 3 was used to prevent calcium-mediated DNA absorption to the lipid bilayer. ${ }^{42}$

\section{Coating CSLBs with DNA for self-assembly}

After the particles were coated with a lipid bilayer using method 2 , various amounts of inert and/or linker DNA were added and the dispersion was gently rotated for $1 \mathrm{~h}$. To remove any remaining free DNA strands in solution, the particles were washed by centrifugation for $2 \mathrm{~min}$ at $43 \mathrm{rcf}$, or alternatively, by sedimentation by gravity for $30 \mathrm{~min}$, and the supernatant was replaced three times by HEPES buffer type 3 .

We define the amount of dsDNA that we add as a surface density $\sigma_{\mathrm{DNA}}$,

$$
\sigma_{\mathrm{DNA}}=\frac{N_{\mathrm{DNA}}}{A_{\mathrm{CSLB}}},
$$

where $N_{\text {DNA }}$ is the total number of dsDNA strands and $A_{\mathrm{CSLB}}$ is the total surface area of the CSLBs. The total number of dsDNA 
strands and particles were estimated from the reported stock concentrations. In this calculation, we assume that all the added dsDNA strands are distributed homogeneously over all particles and that no dsDNA remains in solution. We typically used $\sigma_{\mathrm{DNA}}=320 \mu^{-2}$ dsDNA linkers to obtain flexible structures.

Particle clusters were formed by mixing two particle types coated with complementary DNA linkers in a 1:1 number ratio in a round metal sample holder on a polyacrylamide coated cover slip (see ref. 43 for details). The polyacrylamide coating keeps the particles from sticking to the glass surface, allowing them to cluster via diffusion limited aggregation.

\section{Sample characterization}

The samples were imaged with an inverted confocal microscope (Nikon Ti-E) equipped with a Nikon A1R confocal scanhead with galvano and resonant scanning mirrors. A $100 \times$ oil immersion objective (NA $=1.49$ ) was used. A $561 \mathrm{~nm}$ laser was employed to excite the Lissamine rhodamine dye, a $488 \mathrm{~nm}$ laser was used to excite the TopFluor-Cholesterol dye. The excitation light passed through a quarter wave plate to prevent polarization of the dyes. 500-550 $\mathrm{nm}$ and 565-625 $\mathrm{nm}$ filters were used to separate the emitted light from the TopFluor and the rhodamine dyes, respectively.

The charge of the particles in MilliQ water and HEPES buffer type 1 was determined via zeta potential measurements using a Malvern Zetasizer Nano ZS.

\section{Fluorescence recovery after photobleaching (FRAP)}

We used fluorescence recovery after photobleaching (FRAP) to check the mobility of the lipids in a CSLB. A circular area of the fluorescent sample was bleached, the recovery signal was collected and normalized as

$$
I_{\text {corr }}(t)=\frac{I(t)}{I(t=0) I_{\mathrm{ref}}(t)},
$$

where $I_{\text {corr }}(t)$ is the measured intensity $I(t)$ normalized with respect to the intensity just before bleaching $I(t=0)$ and corrected for bleaching through measurement of the intensity of a non-bleached reference area, $I_{\text {ref }}(t)$. Additionally, we subtracted the background signal from $I$ and $I_{\text {ref }}$. We found that the signal can be fitted using the following expression:

$$
I_{\text {corr }}(t)=A\left(1-\mathrm{e}^{-\frac{t-t_{0}}{\tau}}\right)
$$

where $A$ is the extent of the recovery, $t-t_{0}$ is the time elapsed since the beginning of the recovery process and $\tau$ the recovery time.

While there is a simple relation linking $\tau$ to $D$ for circular bleaching areas on planar surfaces, ${ }^{44}$ we are not aware of a similar expression for a spherical surface that is partly bleached from the side, as is the case in our experiments. Therefore, we quantify the lateral mobility in terms of the recovery time $\tau$ only.

All FRAP experiments on silica particles were performed using $7 \mu \mathrm{m}$ particles, unless stated otherwise. To measure the lateral mobility of DNA linkers using FRAP, no fluorescently labeled lipids were used and instead, we used a high linker

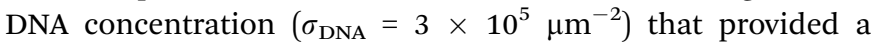
sufficiently bright fluorescent signal.

\section{Particle stability analysis}

To estimate the colloidal stability of particles, we rotated the particles $(0.4 \% \mathrm{w} / \mathrm{w})$ in a test tube for at least $1 \mathrm{~h}$, thereby allowing them to aggregate. We then immobilized some of the clusters on a glass substrate, allowing us to take a "snapshot" of the cluster distribution at that time. Particles were located in bright-field microscopy images of these sedimented, semidilute (volume fraction $\phi \approx 0.001$ ) samples. The cluster sizes were determined by using the bandpass, locate and cluster functions from TrackPy. ${ }^{45}$ Erroneously tracked positions were corrected manually. The separation distance below which particles are considered to be part of a cluster was chosen to be $1.1 D$, where $D$ is the particle diameter. This can lead to a small overestimation of the number of clusters when particles are close together but have not irreversibly aggregated. We defined the fraction of single particles $f_{\text {single }}$ as the number of detected clusters with a size of 1 (i.e. single particles) divided by the total number of individual particles. The error on this fraction was estimated as the standard deviation of the average cluster size divided by the square root of the total number of particles. For each measurement, we analyzed between 150 to 4000 individual particles.

\section{Trimer flexibility analysis}

We have analyzed three linearly-connected CSLBs that were functionalized with inert dsDNA and linker dsDNA. To quantify the mobility of the self-assembled trimers, we tracked the position of the three individual particles in bright-field movies as a function of time and calculated the opening angle $\theta$ between them. For tracking and calculating $\theta$, we used a custom algorithm that is depicted in Fig. S2 of the ESI. $\dagger$

First, the user selects the particles of interest from the first frame (see Fig. S2A, ESI $\dagger$ ). This increases the computational efficiency of tracking because it reduces the number of tracked features and allows for cropping of all frames. We then iterate over all frames to identify the positions of the selected particles. Each current frame is inverted, so that all particles have a ring of high intensity around them (see Fig. S2B, ESI $\dagger$ ). The frame is converted to polar coordinates with the current provisional particle position at the origin (see Fig. S2C, ESI $\dagger$ ), where the provisional position is the one that the user selected for the first frame and the previous tracked position for all subsequent frames. For each row (each polar angle), the position of maximum intensity is found (see Fig. S2D, ESI $\dagger$ ) and these coordinates are then converted back to the original Cartesian coordinate system of the frame (see Fig. S2E, ESI $\dagger$ ). A circle is fitted to these coordinates using a least squares method (see Fig. S2F, ESI $\dagger$ ). After all three particles are found in this frame, the opening angle between them is determined using simple trigonometry (see Fig. S2G, ESI $\dagger$ ). From the opening angles of all the frames, we calculated the 
mean squared displacement of the angle (MSAD or "joint flexibility" $J) .^{16}$

We analyzed the free energy of trimers as function of opening angle using two methods: (1) by converting the histogram to the free energy using Boltzmann weighing and (2) using a maximum likelihood estimation method of angular displacements. ${ }^{43,46}$ We confirmed that both methods agreed and show only the result of the maximum likelihood method, because it allows us to estimate the error in our measurement. We now describe these methods in detail.

Trimer free energy: Boltzmann weighing. We obtained a histogram of opening angles between $60^{\circ}$ to $300^{\circ}$ with a bin width of $3^{\circ}$. We then mirrored and averaged the data around $180^{\circ}$ and converted this to a probability density function. From the probability density function we determined the free energy using Boltzmann weighing,

$$
\frac{F}{k_{\mathrm{B}} T}=-\ln P+\frac{F_{0}}{k_{\mathrm{B}} T},
$$

where $F$ is the free energy, $k_{\mathrm{B}}$ is the Boltzmann constant, $T$ is the temperature, $P$ is the probability density and $F_{0}$ is a constant offset to the free energy, which we chose at a reference point $\left(180^{\circ}\right)$ so that the free energy is equal to zero there.

Trimer free energy: maximum likelihood estimation. While the Boltzmann weighing method is very straightforward, it gives no information about the experimental error. To estimate the error, we used an analysis that is based on a maximum likelihood method in which particle displacements are modelled, ${ }^{43,46}$ which we adapted for our experimental system. We used (angular) displacements because for Brownian particles they are uncorrelated in time, in contrast to positions (or values of the opening angle). This means that using this method, we can obtain reliable results even for a limited number of particles. ${ }^{47}$

To summarize, we followed the method outlined in Chapter 3.4.2 of ref. 46: we find the maximum likelihood estimate of the local force field $F(\theta)$ by using a model for the transition probability $P$ :

$$
P\left(\theta_{1}, t+\tau \mid \theta_{0}, t\right)=(4 \pi D \tau)^{-\frac{1}{2}} \exp \left(-\frac{(\Delta \theta-\beta D F(\theta) \tau)^{2}}{4 \pi D \tau}\right)
$$

where $\theta_{0}$ is the opening angle at a time $t$ and $\theta_{1}$ is the angle at a later time $t+\tau, \tau$ is the time between measurements, $D$ is the diffusion coefficient determined from the mean squared displacement, $\Delta \theta=\theta_{1}-\theta_{0}$ and $\beta$ is the inverse of the Boltzmann constant times the temperature. A Bayesian method was used to find the maximum likelihood estimate by using emcee $^{48}$ and the error was determined as the standard deviation of the chain of Markov Chain Monte Carlo (MCMC) samples. We determined the free energy up to an arbitrary choice of a reference energy by numerical integration of this force. This free energy was then mirrored around $180^{\circ}$ and averaged to determine the free energy between $60^{\circ}$ and $180^{\circ}$. We observed a boundary effect inherent to the analysis for angles smaller than $60^{\circ}+\sqrt{2 J \tau}$ (where $J$ is the joint flexibility) leading to a slight overestimation of the free energy for those angles.

\section{Results and discussion}

We will now characterize every step in the formation of CSLBs. In the first section of the results we study the homogeneity and mobility of the lipid bilayer on colloidal particles made from different materials, and therefore various surface functionalities and degrees of roughness. Furthermore, we investigate the effect of PEGylated lipids on the homogeneity and mobility of the lipid bilayer and their use as steric stabilisers to prevent nonspecific aggregation.

Having found conditions that yield colloidal particles with a homogeneous and mobile bilayer, we subsequently introduce double-stranded DNA connected to a hydrophobic anchor into the bilayer, as shown in Fig. 1(2). We employ DNA constructs both with and without single-stranded sticky ends, to investigate their use in DNA-mediated binding and their effect on colloidal stability, respectively. Finally, we demonstrate that CSLBs can be used for self-assembly by employing DNA linkers with complementary single-stranded sequences. We use FRAP to measure the lateral mobility of DNA linkers on the particle surface inside and outside the bond area. In this way, we show that they are mobile if the bilayer is fluid and that, in this case, the particles can freely roll over each other's surfaces when bonded.

\section{Lipid bilayer coating of colloidal particles}

To use CSLBs in self-assembly studies or as model membrane systems, it is critical that a homogeneous and fluid bilayer forms on the colloidal particles. This implies successful assembly of both leaflets of the bilayer and lateral mobility of the lipids, and hence proteins, linkers, and larger lipid domains, in the membrane. The formation of lipid bilayers on solid supports can be achieved by deposition of SUVs under physiological conditions ${ }^{49}$ as shown in Fig. 1(1). A combination of electrostatic and van der Waals forces between the SUVs and the surface of the support lead to spreading of the liposomes, which, because of hydrophobic/hydrophilic interactions, fuse together on the substrate. ${ }^{50-53}$ Between the surface of the support and the bilayer a thin layer of water remains, allowing the lipids to laterally diffuse in the absence of other motionrestricting forces. Previous studies on planar supported lipid bilayers (SLBs) reported that there are many factors which can prevent homogeneity and mobility of the bilayer. ${ }^{54}$ These factors are related to the surface that is coated (its surface charge, chemical composition and roughness), the aqueous medium ( $\mathrm{pH}$ and ionic strength), the SUVs (composition, charge, size, hydrophobic/hydrophilic balance, transition temperature) and the temperature at which the lipid coating happens. ${ }^{50,55}$ Here we will study how some of these factors, that are inherent to the use of solid particles, influence the formation of supported lipid bilayers on colloidal substrates.

Influence of the chemical properties of the particle surface. The available variety of colloids with anisotropic shapes makes 
1)
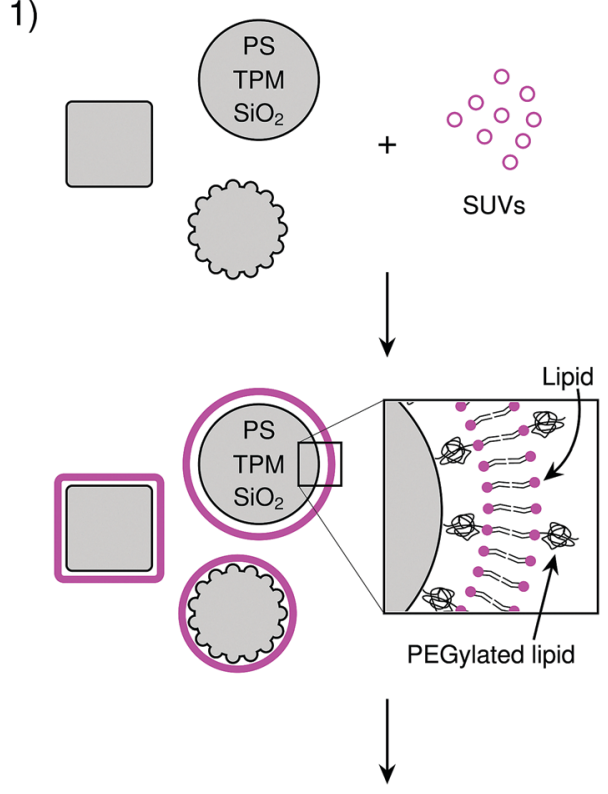

2)

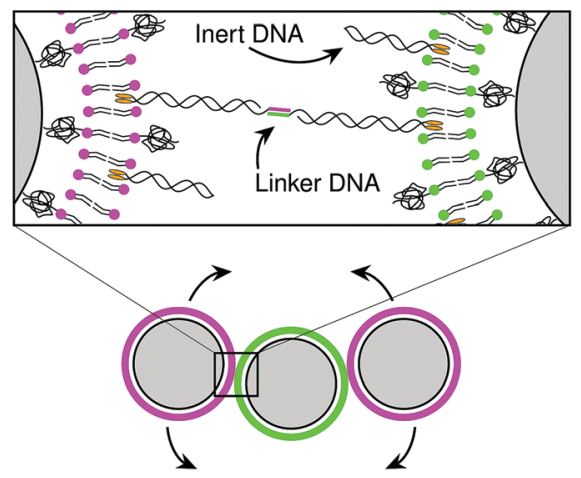

Fig. 1 Overview of the experimental system. (step 1) Micrometer sized colloidal particles are coated with a lipid bilayer by adding small unilamellar vesicles (SUVs) that rupture and spread on the particle surface. We varied the composition of the lipids, as well as the material and shape of the particles. (step 2) DNA linkers with hydrophobic anchors can be added to make particles that are functionalized with DNA with complementary sticky ends. When the lipid bilayer is fluid, the linkers can diffuse over the particle surface and therefore also the linked particles can slide over each other.

them attractive for self-assembly and model membrane studies. Current synthetic procedures tailored to obtain different shapes, however, typically rely on the use of specific materials and therefore yield colloids with different surface properties. We have selected a range of particles of different shapes and commonly used materials to test for membrane homogeneity and mobility after coating with SUVs. In particular, we tested silica spheres prepared by a sol-gel method, commercially available polystyrene spheres, polystyrene spheres with carboxyl groups made using a surfactant-free dispersion polymerization method, ${ }^{40} 3$-(trimethoxysilyl)propyl methacrylate (TPM) spheres, ${ }^{38}$ TPM spheres functionalized with carboxyl and amino groups, ${ }^{39}$ silica-coated polystyrene-TPM spheres and symmetric and asymmetric dumbbells; ${ }^{11}$ as well as hematite cubes ${ }^{35}$ and silica-coated hematite particles. ${ }^{36}$ Silica-coated polystyrene-TPM dumbbells and hematite cubes were obtained by depositing a silica layer following the Stöber method. ${ }^{56}$

After coating, we visually inspect the lipid-coated particles using confocal microscopy and consider bilayers to be homogenous if more than $50 \%$ of the particles do not show strong variations of the fluorescence intensity signal on individual colloids, which we associate to defects of the bilayer. We characterize the bilayer fluidity by measuring the mobility of the fluorescently-labeled lipids on the colloid surface using FRAP. After bleaching, we observe the recovery of the fluorescence intensity due to the diffusion of the dyed lipids in and out of the bleached area. We consider the lipids and thus the bilayer to be mobile if the intensity signal recovers homogeneously in the bleached area, otherwise we consider them to be (partially) pinned to the surface.

Our first observation was that only particles that possess a silica surface exhibited homogeneous and mobile bilayers (Table 1). For spherical silica particles, the fluorescence intensity was homogeneous (see Fig. S1 in the ESI $\dagger$ ), which indicates that all particles are successfully coated with a homogeneous bilayer. We did not succeed in coating colloids made from polystyrene or hematite with a homogeneous bilayer. However, once such substrates were first coated with a silica shell, the bilayer was found to be both homogeneous and mobile. Unexpectedly, particles made from an organosilica compound (TPM) whose surfaces are similar to silica ${ }^{38}$ only showed homogeneous, but not mobile bilayers. Since silica, TPM and polystyrene colloids were all negatively charged (see Table 1), we conclude that the chemical composition of the substrate and not only the surface charge plays a fundamental role in the homogeneity and fluidity of the bilayer. These results agree with previous experiments on

Table 1 Classification of bilayer spreading and lipid mobility based on the material and the surface charge of the colloidal substrates. All zeta potential measurements were performed in MilliQ water and HEPES buffer type 1 at room temperature

\begin{tabular}{|c|c|c|c|c|c|}
\hline \multirow[b]{2}{*}{ Type } & \multirow[b]{2}{*}{ Material } & \multicolumn{2}{|c|}{ Zeta potential $[\mathrm{mV}]$} & \multirow[b]{2}{*}{ Bilayer homogeneity } & \multirow[b]{2}{*}{ Bilayer mobility } \\
\hline & & Water & Buffer & & \\
\hline 1 & Silica spheres (Stöber method, microparticles $\mathrm{GmbH}$ ) & $-56 \pm 6$ & $-23 \pm 2$ & Yes & Yes \\
\hline 2 & Hematite cubic particles ${ }^{35}$ & $+39 \pm 5$ & $+12 \pm 1$ & No & No \\
\hline 3 & Silica-coated hematite cubic particles ${ }^{36}$ & $-32 \pm 6$ & $-6 \pm 2$ & Yes & Yes \\
\hline 4 & Polystyrene spheres (Sigma Aldrich) & $-38 \pm 2$ & $-34 \pm 3$ & No & No \\
\hline 5 & Polystyrene spheres with carboxyl groups ${ }^{40}$ & $-43 \pm 1$ & $-43 \pm 1$ & No & No \\
\hline 6 & Silica-coated polystyrene-TPM (an)isotropic particles ${ }^{11,37}$ & $-33 \pm 1$ & $-21 \pm 2$ & Yes & Yes \\
\hline 7 & TPM spheres $^{38}$ & $-42 \pm 1$ & $-20 \pm 1$ & Yes & No \\
\hline 8 & TPM spheres with carboxyl groups ${ }^{39}$ & $-46 \pm 1$ & $-23 \pm 2$ & No & No \\
\hline 9 & TPM spheres with amino groups ${ }^{39}$ & $-12 \pm 4$ & $-17 \pm 1$ & No & No \\
\hline
\end{tabular}


planar SLBs, in which silica-based surfaces were found to be one of the most reliable supports for homogeneous and mobile lipid bilayers. $^{50}$

Since colloidal particles are often functionalized with different groups on the surface, we furthermore have studied the bilayer on particles equipped with surface groups commonly used in colloidal science (Table 1). While TPM particles with an unmodified silica-like surface showed homogeneous bilayers, we found that functionalization with negatively charged carboxyl or positively charged amino groups prevented spreading and fusion of the lipid vesicles. Likewise, functionalization of polystyrene spheres with carboxyl groups did not enhance the homogeneity of the lipid bilayer. A previous study on planar SLBs reported that the spreading of SUVs depends on the combination of the molecular ions in the buffer and the type and density of surface charge. ${ }^{57}$ While amino-functionalized surfaces are hence expected to disrupt the spreading of SUVs in the presence of the negatively charged HEPES molecules of the buffer, the observation of inhomogenous bilayers on carboxylfunctionalized surfaces can likely be allocated to an insufficiently dense surface coverage. We conclude that similar to planar SLBs, the homogeneity and fluidity of the bilayer of CSLBs depends on a complex interplay of the chemical and physical properties of the lipids and the particle's surface.

Influence of particle curvature differences. Another factor that may influence the successful formation of a homogeneous and mobile bilayer is the variation in curvature of the colloidal substrate, which may hinder spreading and fusion of SUVs. Curvature differences can originate from the overall anisotropic shape of the particles or from surface roughness. As discussed before, we found that particles with a comparably smoothly varying curvature, such as hematite cubes or symmetric and asymmetric dumbbells (see Table 1), had a fluid and homogeneous bilayer after coating, if they featured a silica surface clean of any polymer residues from synthesis. Particles with rough surfaces however have a much higher and frequent variation in curvature. To investigate the effect of large curvature differences, we prepared two batches of polystyrene particles which only differed in their surface roughness and coated them with a silica layer following a Stöber method. ${ }^{37}$ In Fig. 2 we show that particles with low degree of roughness (A) can be homogeneously coated with a bilayer (B) while particles with very rough surfaces (C) show an inhomogeneous bilayer (D). FRAP experiments confirmed that the bilayer on the particle with lower degree of roughness is not only homogeneous, but also mobile, while the inhomogeneous bilayer on the rougher particle is immobile as indicated by the non-recovering intensity signal. We conclude that the roughness of the surface plays an important role in both bilayer homogeneity and mobility.

Influence of free and grafted polymers. Polymers or surfactants are often employed to stabilize colloidal particles in solution, ${ }^{58-60}$ but may influence the formation and mobility of the bilayer in CSLBs. Here, we test how the presence of, for example, leftover polymers from particle synthesis, affects the bilayer. We compare a sample of silica-coated hematite cubes with and without PVP, a polymer commonly used to achieve stability during the synthesis and long-term storage of the sample. To remove the PVP from the surface after synthesis, we calcinated the colloids at $500{ }^{\circ} \mathrm{C}$ for 2 h. Fig. 3 shows that cubes with PVP possess an inhomogeneous bilayer and the ones without it feature a bilayer that homogeneously covers the surface (Fig. 3B). As expected for Stöber silica surfaces, the bilayer on the colloids for which the PVP was removed is also mobile, as indicated by the recovery of the fluorescence intensity.

Moreover, the presence of polymers may not only affect the bilayer's properties, but also the incorporation of functional groups such as DNA linkers into it. We tested this by preparing CSLBs with fluorescently labeled DNA linkers connected to double cholesterol anchors and transferring an aliquot of this dispersion to a HEPES solution containing $5 \% \mathrm{w} / \mathrm{w}$ of Pluronic F-127, a polymer that is commonly used for the stabilization of
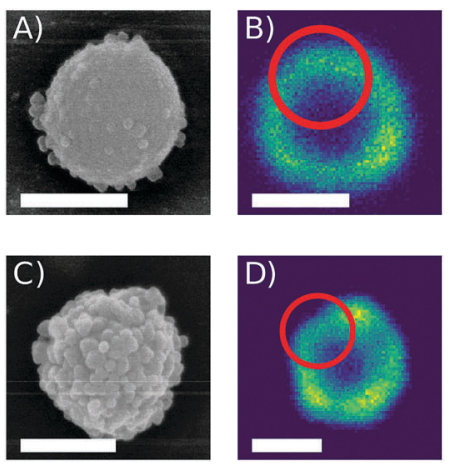

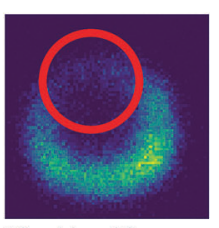

After bleaching

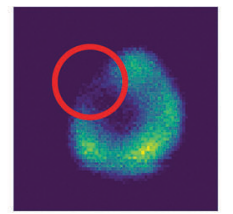

After bleaching

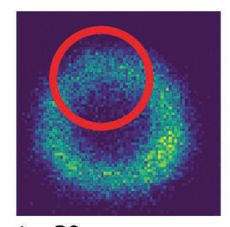

$t=30 \mathrm{~s}$

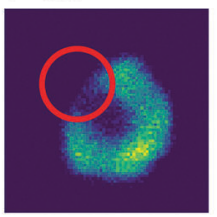

$t=77 \mathrm{~s}$

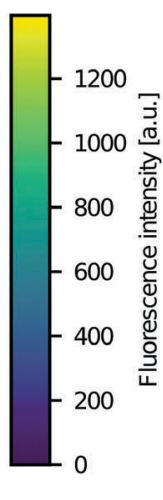

E)

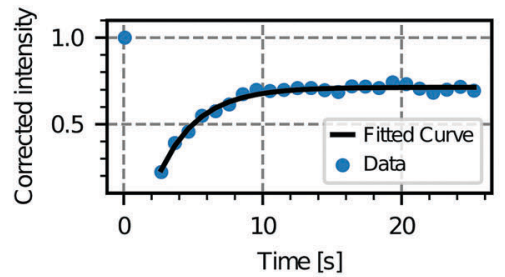

F)

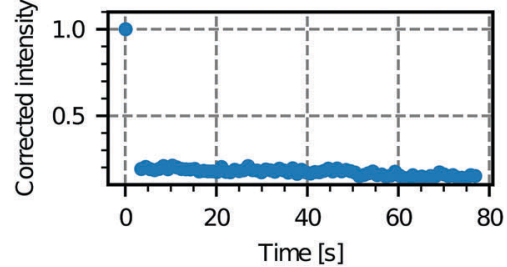

Fig. 2 Effect of surface roughness on lipid bilayer formation. Scanning electron microscopy (SEM) images of particles of type 6 (see Table 1), showing low (A) and high (C) degrees of roughness. Sequence of a FRAP experiment before bleaching, just after bleaching and after $30 \mathrm{~s}$ and $77 \mathrm{~s}$ for the (B) less rough and (D) rougher particle, respectively. On the right, the fluorescence intensity as a function of time and an exponential fit of the data for the (E) less rough and (F) rougher particle are shown. The fluorescence recovery of the bilayer on the smooth particle shows that the bilayer is fluid, in contrast to the rough particle which does not show a recovery of the fluorescence. Scale bars are $1 \mu \mathrm{m}$. 

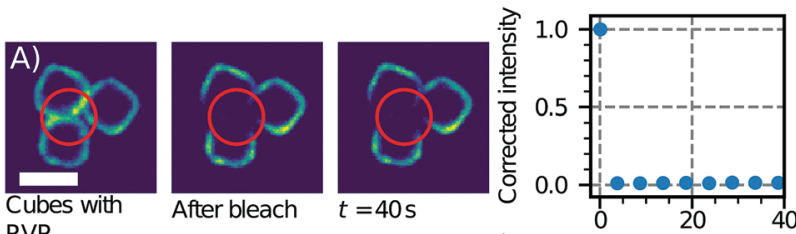

PVP

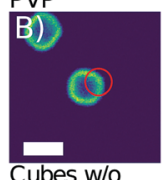

After bleach
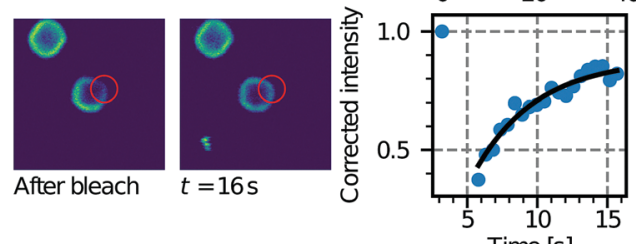

PVP
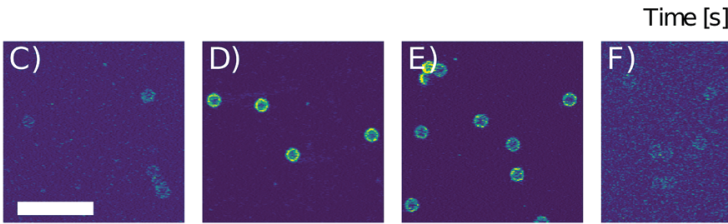

No DNA DNA

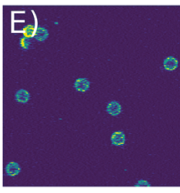

DNA + F-127 (15 min)

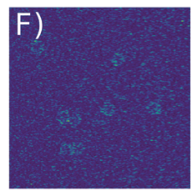

DNA +

F-127 (1 h)

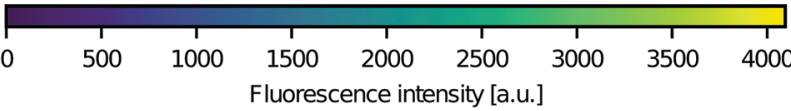

Fig. 3 Effect of PVP on CSLB formation. FRAP experiment on a group of three cubes with (A) and without (B) PVP (particle type 3 without the hematite core, Table 1). Only the sample without PVP shows recovery of the signal in the bleached area. Scale bars are $2 \mu \mathrm{m}$. Influence of F-127 on linker inclusion. (C) Control image of the fluorescence of the undyed CSLBs (particle type 1, Table 1). (D) Fluorescence intensity of CSLBs coated with $20 \mathrm{~nm}$ linker dsDNA. (E) The same sample as in (D), but imaged $15 \mathrm{~min}$ after dispersion in 5\% F-127 solution. The fluorescence on the particles was found to be significantly less homogeneous than in (D). (F) The same sample as in (D), but imaged $1 \mathrm{~h}$ after dispersion in 5\% F-127 solution. The fluorescence intensity is comparable to the uncoated control in (C) so we conclude that all dsDNA has been removed from the bilayer by F-127. Scale bar is $10 \mu \mathrm{m}$.

colloidal particles. While the fluorescent signal of the CSLBs with and without F-127 were initially equal, already 15 minutes after mixing we observed less dsDNA fluorescence on the CSLBS with F-127 compared to particles without it. After $1 \mathrm{~h}$, the fluorescence intensity of the CSLBs with F-127 was comparable to that of control particles not coated with linker dsDNA (Fig. 3C-F). We therefore conclude that F-127 removed the cholesterol-anchored linker DNA from the bilayer, in line with recent experiments on emulsion droplets coated with mobile DNA linkers. ${ }^{61}$

Influence of PEGylated lipids on bilayer homogeneity and mobility. The presence of polymers in SLBs is not always detrimental, but may even improve bilayer mobility. Previous studies on planar SLBs showed that membranes can be supported by polymers covalently bound to lipids (lipopolymer tethers). ${ }^{62-66}$ Since lipopolymer tethers increase the thickness of the water layer between the solid support and the bilayer ${ }^{62-64}$ they are thought to reduce the friction between the substrate and the bilayer, allowing for higher diffusivity of lipid molecules and linkers.

Inspired by this, we study how a specific lipopolymer tether affects the spreading and the fluidity of the bilayer in CSLBs.
We used the lipopolymer DOPE-PEG, a phospholipid with a covalently bound PEG molecule. We employed PEGylated lipids with three different molecular weights: 2000, 3000 and $5000 \mathrm{~g}$ $\mathrm{mol}^{-1}$ in varying concentrations. It is important to note that PEGylated lipids were introduced in the system during the SUV formation by mixing them with the other lipids. This means that once the bilayer is formed, they are present in both leaflets.

We report in Fig. 4A and B the effect of varying concentration and molecular weight of the lipopolymers on the spreading and the mobility of the bilayer. In the absence of PEGylated lipids, the bilayer on the CSLBs was observed to be fluid. At increased concentrations of DOPE-PEG, the bilayer became inhomogeneous, which indicates insufficient spreading and fusion of the SUVs. This effect appeared at lower molar fractions for lipopolymers with higher molecular weights of the DOPE-PEG. For completeness, we note that a small fraction of particles in samples that are labeled as inhomogeneously coated do exhibit a homogeneous, but nevertheless immobile, bilayer. We believe that the reason for the observed inhomogeneity is two-fold. On the one hand, higher concentrations of lipopolymers lead to an increased steric stabilization, that prevents fusion of the SUVs and hinders the van der Waals interactions between the SUVs and the substrate that aid spreading. On the other hand, PEGylated lipids in the brush state increase the bending rigidity of the SUV membrane, thereby preventing rupture and spreading on the surface. ${ }^{67}$

For fluid membranes, we quantified the mobility of the lipids by calculating the recovery time from FRAP experiments, which is the time it takes a bleached area to recover its fluorescence intensity. We find that the diffusion of the lipids is faster for PEGylated lipids with a lower molecular weight and increases with decreasing amount of the lipopolymers, see Fig. 4C. This latter result agrees with a study performed on planar supported lipid bilayers. ${ }^{62}$ In the presence of lipopolymers, we find the shortest recovery time $(3.2 \pm 0.02 \mathrm{~s})$, e.g. highest diffusivity, for $1 \mathrm{~mol} \%$ of DOPE-PEG(2000). The decrease of the diffusion coefficient with the amount of lipopolymer indicates that the PEGylated lipids are pinned to the surface and in this way hinder the mobility of the other lipids.

We emphasize that the mobility of supported lipid bilayers in presence of polymers is dependent on many factors and one may not extend our results to other types of polymers, lipid bilayers or physiological environments. ${ }^{66}$ The complex interplay between polymers and the chemical properties of the colloidal surface can lead to surprising results. For example, and in contrast to what we reported above, we found that an homogenous bilayer on cubic silica particles could only be obtained by using both PEGylated lipids (DOPE-PEG(5000)) and a negatively charged lipid (GM1). Interestingly, at high concentrations of PEGylated lipids the bilayer is very homogeneous but not mobile (Fig. 4D). This is in contrast to silica spheres coated with the same concentrations of lipopolymers and only zwitterionic lipids, which do not possess a homogeneous bilayer, see Fig. 4A. We indicated this state in which the bilayer is homogeneous, but not fluid, with blue squares in Fig. 4D. A possible origin of this unusual behavior could be the different 

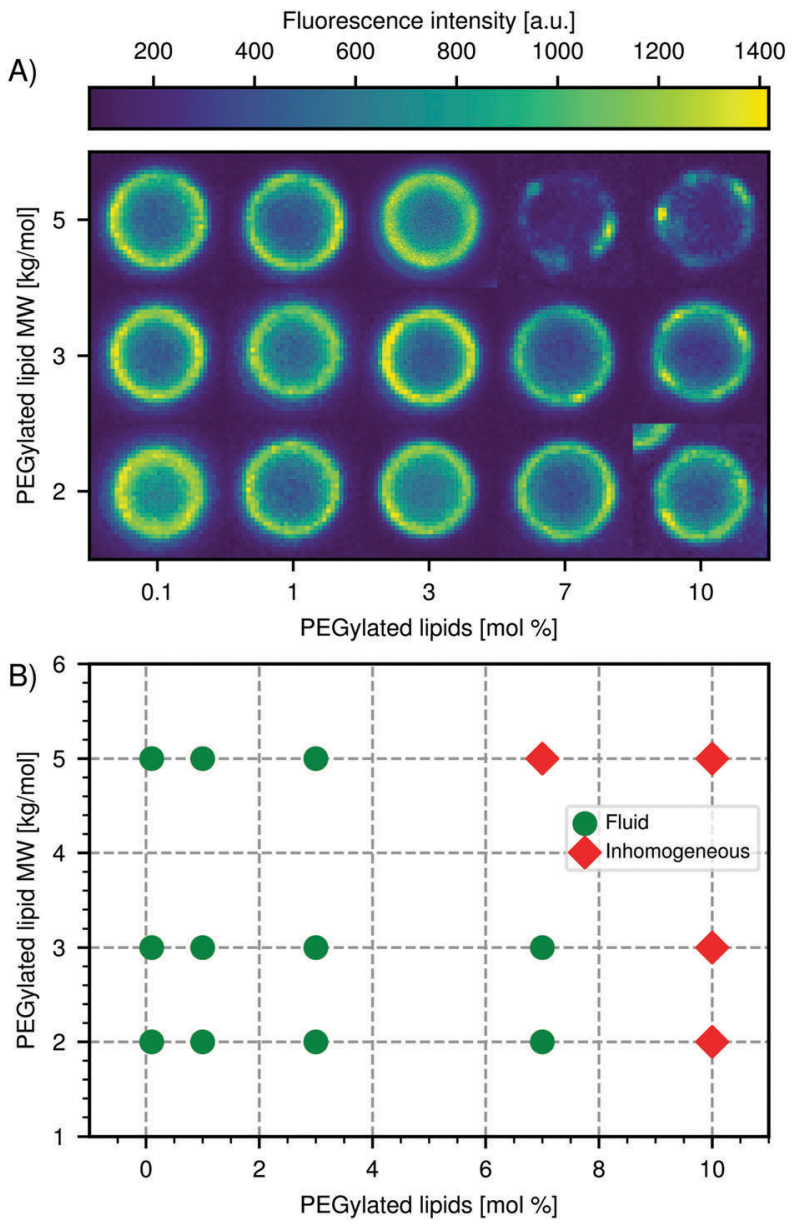
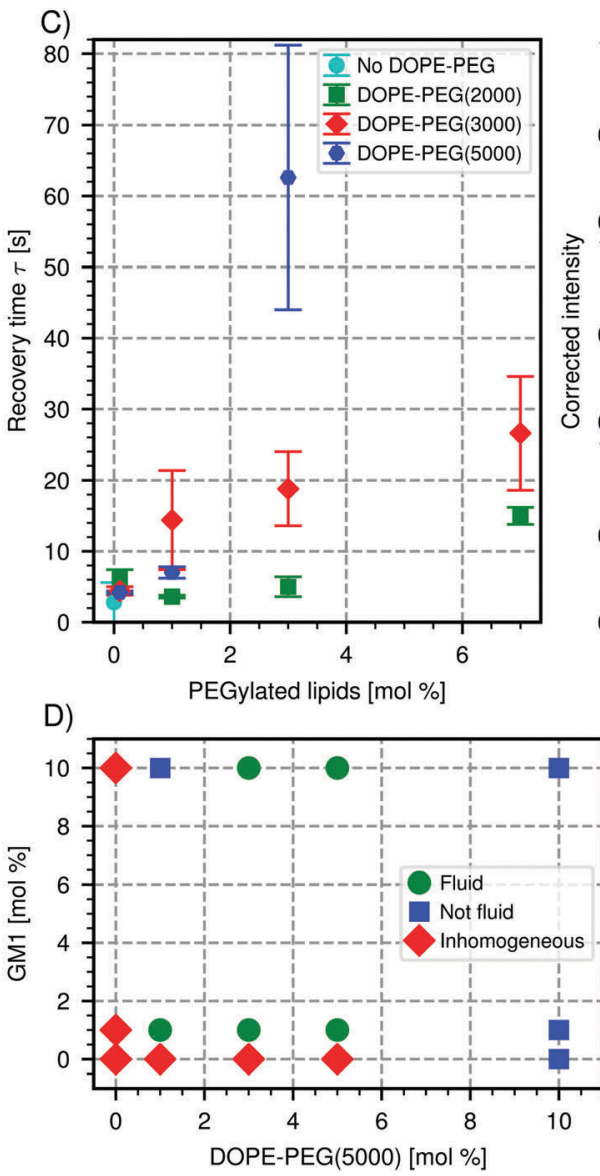
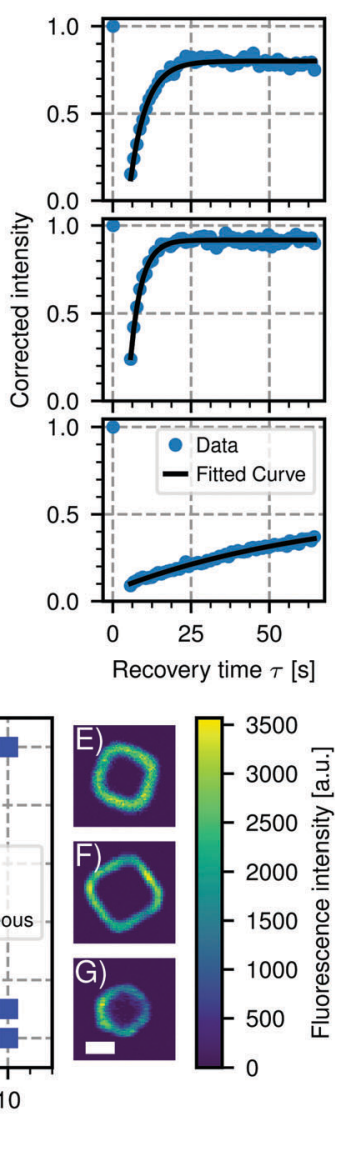

Fig. 4 Effect of PEGylated lipids on CSLB formation and fluidity. (A) Color-map of the intensity of the bilayer of spherical CSLBs (particle type 1, Table 1) ordered by increasing molecular weight and concentration of PEGylated lipids. The images are taken in the equatorial plane. (B) Phase diagram of the state of the bilayer for varying molecular weight and concentration of PEGylated lipids. (C) Left: Plot of the recovery time after FRAP depending on molar concentration and size of the PEGylated lipids. Right: Intensity recovery curves as a function of time from FRAP experiments of, from top to bottom, CSLBs without PEGylated lipids, with 1 mol\% DOPE-PEG(2000) and 3 mol\% DOPE-PEG(5000). (D) Phase diagram of spreading and fluidity of the bilayer on cubic silica shells (particle type 3 without the hematite core, Table 1) depending on GM1 and PEGylated lipids. Colormap of a fluorescent image of a cubic bilayer made with (E) 1 mol\% GM1 and 3 mol\% DOPE-PEG(5000), (F) 1 mol\% GM1 and 10 mol\% DOPE-PEG(5000), (G) no GM1 or DOPE-PEG(5000). Scale bar $1.5 \mu \mathrm{m}$.

porosity, surface chemistry and charge of the silica cubes ${ }^{56}$ compared to the silica spheres (Table 1).

\section{Stabilizing CSLBs against nonspecific aggregation}

To build specific colloidal structures from the bottom up, careful control over the interactions between the particles is required. On the one hand, specific attractive interactions may be employed to control which particles interact. This specific binding can be achieved by using dsDNA linkers with complementary sticky ends. ${ }^{13,16,68}$ On the other hand, the particles need to be prevented from binding to each other nonspecifically: that is, not via dsDNA linker interactions but via other attractive forces that act indiscriminately between all particles, such as van der Waals forces. In other words, it is crucial to be able to control the colloidal stability of the CSLBs. ${ }^{69}$

In our experiments, stabilization by repulsive electrostatic interactions is not a feasible route because surface charges are screened by the counterions in the HEPES buffer that is needed to allow the complementary DNA sticky ends to bind. ${ }^{68}$ The ionic strength of the buffer must be higher than $50 \mathrm{mM}$ so that clusters are formed via DNA-mediated attractions. ${ }^{70}$ At these salt concentrations, even the bare silica particles (particle type 1, Table 1) are no longer stabilized by their negatively charged surface groups. Indeed, we found that both the bare silica particles and the silica particles coated with a lipid bilayer aggregated in all buffers, as was previously observed. ${ }^{71}$ The fraction of single particles determined from light microscopy images was $f_{\text {single }}=0.67 \pm 0.10$ for uncoated silica particles after one hour of mixing in the buffer, while they were previously stable in deionised water. We therefore explored different ways to sterically stabilize the particles using higher concentrations of PEGylated lipids, SDS and inert dsDNA strands.

Stabilization using SDS. SDS is a surfactant with amphiphilic properties, consisting of a polar headgroup and a hydrocarbon tail, that has been shown to stabilize emulsion droplets coated with lipid monolayers. ${ }^{34}$ Inspired by these findings, we added SDS to the CSLBs after bilayer coating to increase their stability. 
However, in contrast to lipid coated emulsion droplets we found no significant increase in stability when we varied the SDS concentration between $0 \mathrm{mM}$ to $1 \mathrm{mM}$. In fact, the highest concentration of $1 \mathrm{mM}$ led to a decrease in particle stability from $f_{\text {single }}=0.67$ without SDS to $f_{\text {single }}=0.45$ at $1 \mathrm{mM}$. This is likely caused by the disruptive effect that SDS can have on lipid bilayers. In a study on large unilamellar vesicles (LUVs) made from POPC, it was found that SDS can completely solubilize the vesicles above concentrations of $2 \mathrm{mM}^{72}$ While this concentration is higher than the concentrations that we used here, we already observed some damage to the bilayer. The resulting inhomogeneous coating may allow nonspecific "sticking" on patches that are not covered with a lipid bilayer and a subsequent decrease in overall particle stability.

Stabilization using PEG. In contrast to SDS, PEGylated lipids can provide colloidal stability through steric repulsions between the PEG moieties while also being easily integrated into the bilayer through their lipid tails. ${ }^{58-60}$ To test their use for colloidal stabilization, we coated the particles with SUVS that contain a small fraction of the following PEGylated lipids: DOPE-PEG(2000), DOPE-PEG(3000) and DOPE-PEG(5000). Since we include these lipopolymers during SUV preparation, they are part of both the inner and outer leaflet, as depicted in Fig. 1(1). At low concentrations, we observed no significant change in the stability of the particles upon an increase in the concentration of PEGylated lipids. For example, for DOPEPEG(2000) the stability remained constant below $5 \mathrm{~mol} \%$ as shown in (Fig. 5A). For concentrations between $1 \mathrm{~mol} \%$ and 7 mol\% for DOPE-PEG(2000), $1 \mathrm{~mol} \%$ and $6.8 \mathrm{~mol} \%$ for DOPEPEG(3000) and $2 \mathrm{~mol} \%$ and $2.7 \mathrm{~mol} \%$ for DOPE-PEG(5000), the average fraction of unclustered particles lay between $f_{\text {single }}=0.5$ and $f_{\text {single }}=0.77$, with no clear trend observed for different polymer lengths or concentrations. For all measurements, we verified that the spreading of the SUVs was successful. We believe that the stability of the particles did not improve significantly because at these concentrations, the grafted PEG was in the mushroom state instead of the brush state, and therefore not sufficient to provide steric stability. ${ }^{73,74}$ Therefore, we increased the concentration of DOPE-PEG(2000) above 7 mol\%. Indeed, as shown in Fig. 5, the colloidal stability increases above this concentration, likely due to a transition from the mushroom to the brush state, as was shown for similar lipopolymers (DSPE-PEG(2000)) around 8 mol\%. ${ }^{75}$

While the colloidal stability can be improved by increasing the concentration of PEGylated lipids, the bilayer is not fluid at the concentrations required for colloidal stability as we showed earlier (see Fig. 4B). Therefore, embedded DNA linkers will also not be mobile in the bilayer and it is not possible to form reconfigurable structures from CSLBs stabilized by PEGylated lipids only.

Stabilization using inert dsDNA. Since PEGylated lipids cannot be used to provide steric stability because they reduce the fluidity of the membrane, we explored an alternative route to stabilize the CSLBs. Inspired by numerical findings that inert double stranded DNA (dsDNA) can also act as a steric stabilizer via excluded volume interactions between DNA strands on different particles, ${ }^{76}$ we employed dsDNA strands with a double
A)

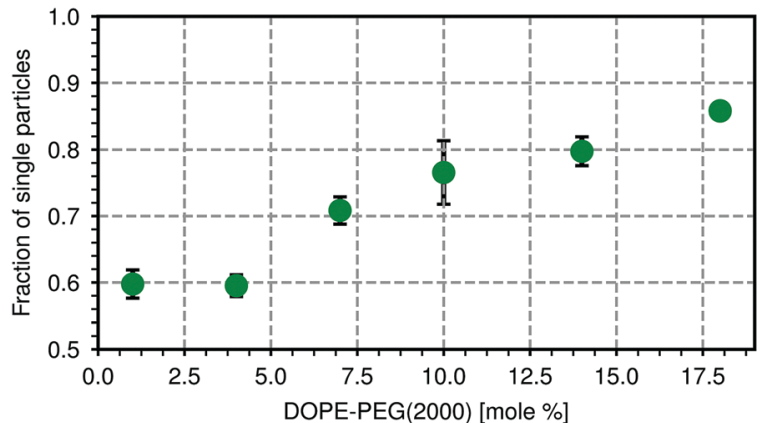

B)

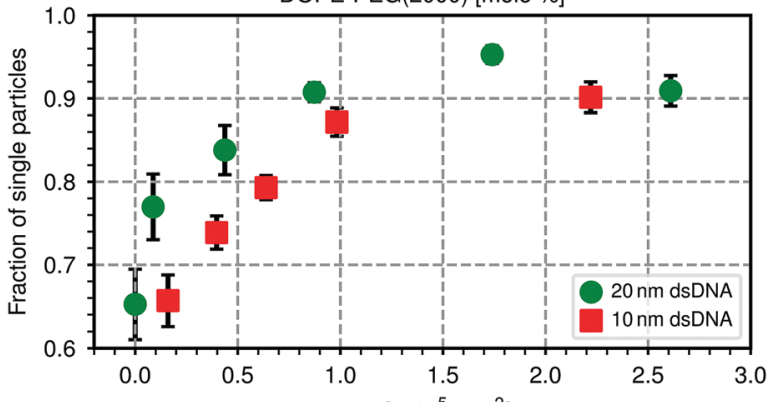

C)

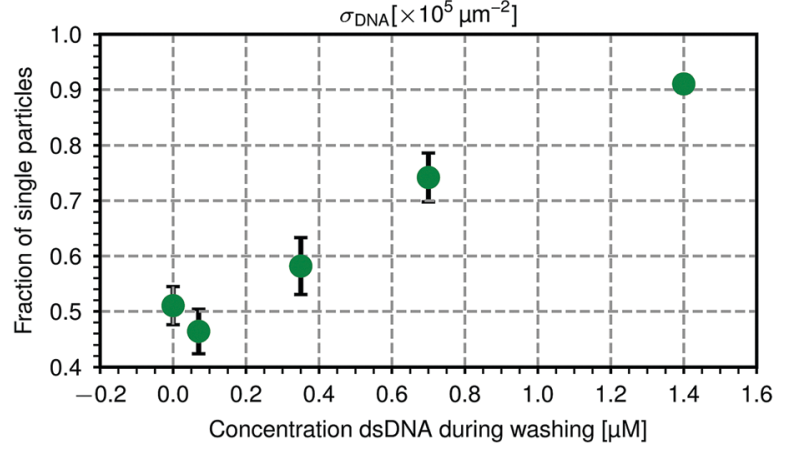

Fig. 5 Steric stabilization of CSLBs. (A) Higher concentrations of DOPEPEG(2000) lead to a higher fraction of single particles in the absence of linker DNA. Stability is shown after one washing cycle of 2 min at $43 \mathrm{rcf}$ and overnight rotation. We hypothesize that above $8 \mathrm{~mol} \%$, the packing density of PEG on the surface of the membrane is high enough for the PEG to be in the brush state, making it more effective as a steric stabilizer. (B) Increasing the number of dsDNA strands on the particle surface increases the particle's stability for two different lengths of inert dsDNA $(10 \mathrm{~nm}$ and $20 \mathrm{~nm}$ ). (C) Centrifugation and redispersion with a solution containing inert dsDNA affects the fraction of single particles. After centrifuging particles that were initially stable $\left(f_{\text {single }}=0.95, \sigma_{\mathrm{DNA}}=1.7 \times 10^{5} \mu \mathrm{m}^{-2} \mathrm{dsDNA}\right) 3 \times$ at 43 rcf for 2 min, we observed nonspecific aggregation $\left(f_{\text {single }}=0.51\right)$ in the absence of dsDNA strands in solution while increasing the concentration of inert dsDNA $(20 \mathrm{~nm})$ in the washing solution could preserve stability. Particles of type 1 (see Table 1) was used in all experiments.

cholesterol anchor at one end to functionalize the CSLBs with this DNA via hydrophobic insertion of the cholesterol into the bilayer, ${ }^{14}$ see Fig. 1(2). Because the cholesterol anchors insert into the hydrophobic core of the bilayer, which acts as an energetic barrier to the highly charged DNA molecules, ${ }^{42}$ the DNA is confined to the outer leaflet.

We varied the surface concentration $\sigma_{\text {DNA }}$ (see eqn (1)) of two inert dsDNA strands with different lengths and measured its effect on the particle stability, which is shown in Fig. 5(B). The stability was determined after the particles were coated with inert dsDNA and rotated for one hour. For both the $10 \mathrm{~nm}$ inert 
dsDNA and the $20 \mathrm{~nm}$ inert dsDNA, we found that increasing the number of grafted dsDNA strands per particle led to an increase in particle stability from $f_{\text {single }}=0.68$ without dsDNA to $f_{\text {single }}=0.90$ to 0.95 above $\sigma_{\mathrm{DNA}}=2 \times 10^{5} \mu \mathrm{m}^{-2}$. We found that the $20 \mathrm{~nm}$ inert dsDNA is slightly more efficient as a steric stabilizer than the $10 \mathrm{~nm}$ inert dsDNA, as can be seen in Fig. 5(B). This is expected, because for the longer DNA, excluded volume interactions between particles start to become important already at larger particle separations than for the shorter DNA. Additionally, excluded volume interactions between DNA strands on the same particle force the DNA to extend outwards already at lower concentrations for the longer DNA strands as compared to the shorter DNA strands. Therefore, the repulsion between the particles also has a longer range, leading to better colloidal stability. However, at concentrations above $\sigma_{\mathrm{DNA}}=2 \times 10^{5} \mu \mathrm{m}^{-2}$, the difference between the $10 \mathrm{~nm}$ and $20 \mathrm{~nm}$ inert dsDNA is less pronounced, because the particles are so densely coated that adding longer or more DNA strands will not stabilize the particles any further.

To use these particles in self-assembly studies, specific interactions need to be present as well, which we here induce by adding dsDNA linkers, that is, dsDNA strands with a sticky end and double cholesterol anchors. After the particles are functionalised, any excess linker DNA left in the solvent needs to be removed to reduce unwanted aggregation or saturation of the complementarily functionalized particles. To remove excess linker DNA, we washed and redispersed the particles in buffer solution three times and measured the particle stability afterwards. Unexpectedly, it decreased from $f_{\text {single }}=0.95$ before washing to $f_{\text {single }}=0.51$ after washing. To detect whether the partial removal of the stabilizing inert dsDNA during washing had caused this aggregation, we washed the particles in a HEPES buffer that contained various concentrations of inert dsDNA. As shown in Fig. 5(C), increasing the concentration of inert dsDNA in the washing solution led to an increase in particle stability after washing, therefore confirming our hypothesis. Including 1.4 $\mu \mathrm{M}$ of inert DNA in the washing solution allowed us to preserve the particle stability $\left(f_{\text {single }}=0.91\right)$.

However, washing the particles with such high concentrations of inert dsDNA proved detrimental to the bilayer and led to the formation of membrane tubes between $1 \mu \mathrm{m}$ to $3 \mu \mathrm{m}$ long (see Fig. S4 in the ESI $\dagger$ ). These membrane tubes are highly curved surfaces that are only formed under specific conditions, for example a difference in spontaneous curvature between the inner and the outer membrane leaflets. ${ }^{77}$ In our case, since dsDNA is only added after the formation of the bilayer it is only present in the outer leaflet and hence induces a difference in spontaneous curvature which causes formation of tubes. If the DNA would be present on both leaflets, no tube formation is expected. We tested this by mixing the dsDNA with the SUVs before coating the particles but found no fluorescence signal on the particles' surface, implying the absence of a bilayer coating (see Fig. S5 in the ESI $\dagger$ ). We believe that the dsDNA sterically stabilizes the SUVs and prevents spreading and fusion of SUVs on the particle surface.

To summarize, we found that inert double-stranded DNA can impart colloidal stability to the CSLBs. Asymmetric distribution of DNA causes the formation of membrane tubes

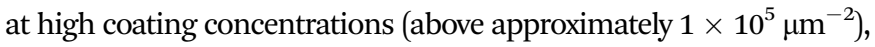
but is unavoidable if colloidal stability needs to be preserved during repeated washing cycles.

\section{Linker functionalization for self-assembly}

To be able to employ CSLBs in self-assembly experiments, we induce specific attractive interactions by using two sets of dsDNA linkers with complementary single-stranded sticky ends that can form bonds via hybridization. We use two hydrophobic anchors (either cholesterol or stearyl) per dsDNA complex to insert the dsDNA linker into the outer leaflet (see Fig. 1(2)), while at the same time adding inert dsDNA strands for steric stabilization. We use double hydrophobic anchors because dsDNA with a single hydrophobic anchor is less strongly confined to the bilayer. ${ }^{14}$ The dsDNA is attached to the double hydrophobic anchor with a tetraethylene glycol (TEG) or hexaethylene glycol (HEG) spacer to allow it to swivel with respect to the anchor. We label the dsDNA linkers with a fluorescent dye to image them using confocal microscopy. Full details on the DNA strands we used can be found in Table S1 of the ESI. $\dagger$

Previous experiments ${ }^{16}$ have shown that several interesting structures, such as flexible colloidal polymers and molecules, can be obtained via self-assembly of CSLBs. In order to form these reconfigurable structures, not only the lipids in the bilayer should be mobile but also the grafted linker DNA should be mobile on the surface of the membrane. We can quantify the mobility of dsDNA on the surface of the bilayer by measuring the FRAP of fluorescently labeled DNA. Note that for these experiments we did not employ fluorescent lipids. For a successful recovery after bleaching of the DNA linkers in the binding patch between two particles, two requirements need to be fulfilled: the DNA linkers outside of the binding patch have to be mobile and the bleached linkers inside the binding patch have to be able to unbind, to allow unbleached linkers to diffuse into the binding patch. We calculate the melting temperature of the sticky end using an approximate formula ${ }^{78}$ and find a melting temperature $T_{\mathrm{m}}=30.4{ }^{\circ} \mathrm{C}$, meaning that at $25{ }^{\circ} \mathrm{C}$ the probability for the sticky end to form a duplex is $P\left(25^{\circ} \mathrm{C}\right)=0.68$ based on melting temperature considerations only. Therefore, the sticky ends continuously bind and unbind in our experiments, making fluorescence recovery possible, while the sheer number of linkers in the patch area keeps the particles always bound.

We confirmed the mobility of linker DNA on the particle surface using FRAP experiments, shown in Fig. 6. Note that the whole cluster is immobilized on the (non-passivated) glass coverslip to enable FRAP on these micrometer-sized particles. Therefore these clusters do not show translational diffusion or cluster flexibility. In Fig. 6(A), a representative FRAP experiment on a single sphere is shown. We measured an average recovery time of $(8 \pm 2)$ s from independent measurements on 16 different particles. Outside the bond area (Fig. 6B), we measured an average recovery time of $(6 \pm 2) \mathrm{s}$ from independent measurements on 15 different clusters. Within the error, the diffusion of DNA outside of 

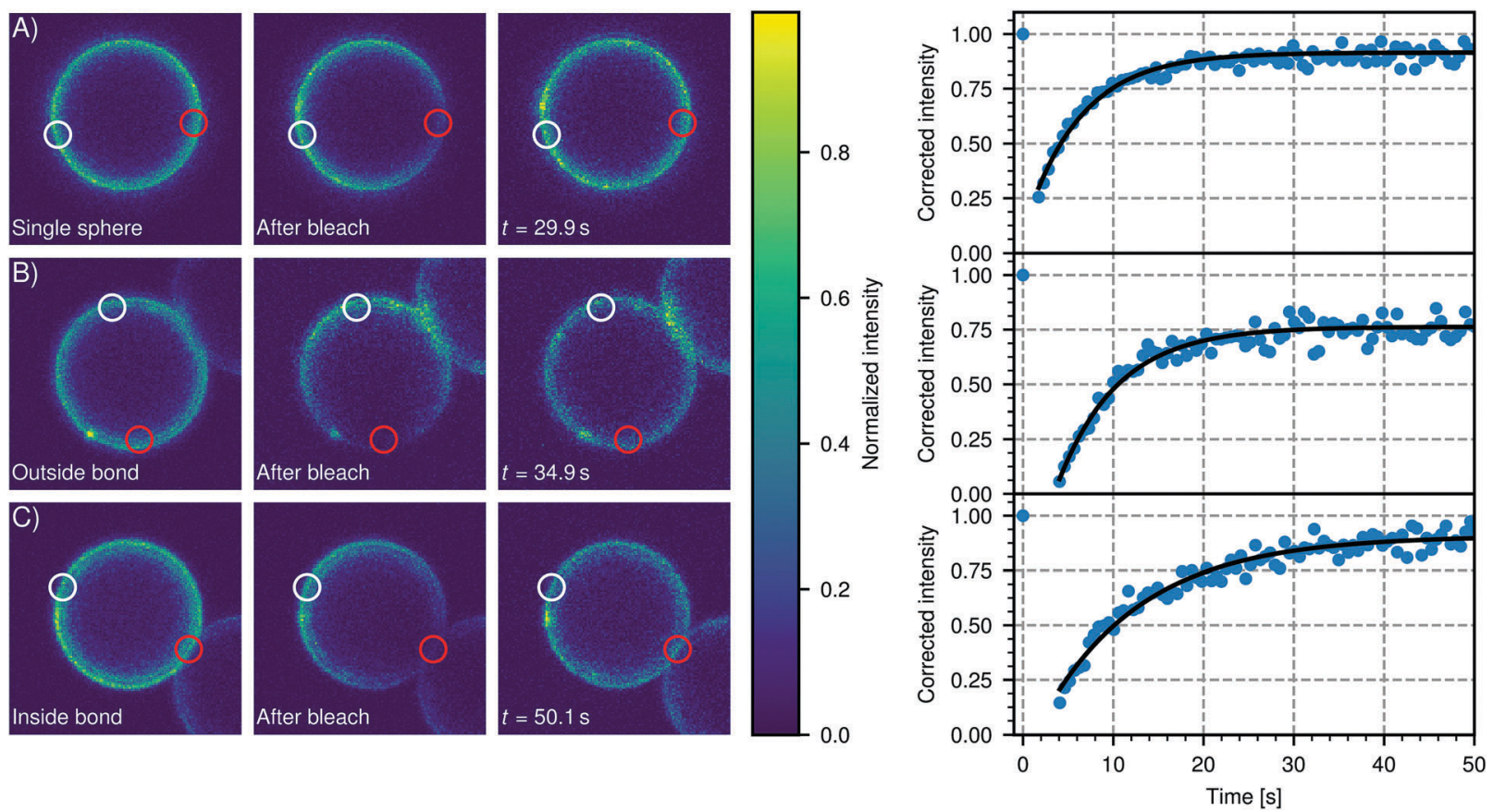

Fig. 6 DNA linker mobility on CSLBs. Three representative FRAP experiments are shown that probe the mobility of DNA linkers on the surface of a $7 \mu$ m CSLB (particle type 1, Table 1). In the intensity color plots, a red circle indicates the bleached area and a white circle shows the reference area. For all experiments, the DNA linkers are mobile. (A) FRAP on a single sphere. We measured an average recovery time of $(8 \pm 2) \mathrm{s}$ from independent measurements on 16 different particles. (B) FRAP on a sphere in a cluster, taken outside the bond area. We measured an average recovery time of (6 \pm 2 ) $\mathrm{s}$ from independent measurements on 15 different clusters. Note that, within the error, this is the same as the recovery time we measured for single particles in (A). (C) FRAP on a sphere in a cluster, inside the bond area. We measured recovery times of $5 \mathrm{~s}$ to $62 \mathrm{~s}$ with an average recovery time of $(17 \pm 16) \mathrm{s}$ from independent measurements on 12 different clusters. Note that this is longer than the recovery times we measured outside the bond area in (A) and (B), which indicates the diffusion is slower in the bond area. However, the spread in recovery times was also larger for bleached areas inside the bond area.

the bond area is the same as on free particles, as expected. Inside the bond area (Fig. 6C), we measured recovery times of $5 \mathrm{~s}$ to $62 \mathrm{~s}$ with an average recovery time of $(17 \pm 16) \mathrm{s}$ from independent measurements on 12 different clusters. This is longer than the recovery times we measured outside the bond area and on single particles, which indicates the diffusion is slower in the bond area. However, the recovery time inside the bond area varied greatly between different clusters indicating that diffusion into and out of the bond area can be different. A likely cause is the spread in the DNA concentration between individual particles in a batch. Higher DNA concentrations imply a higher concentration of bonded linkers in the bond area. This will sterically hinder the diffusion of unbonded linkers inside the patch area and will thus lead to longer recovery times. Furthermore, we hypothesize that the linker concentration in the patch area slowly increases as a function of time after binding, so that the recovery time depends on the time that has elapsed after the formation of the cluster, which we did not control. In summary, we have shown here that the dsDNA linkers are mobile on each part of the (un)bound particle, which is a prerequisite for creating flexibly linked clusters.

\section{Mobility of self-assembled structures}

The mobility of individual dsDNA linkers on the surface does not necessarily imply that bonded clusters of DNA functionalized CSLBs are also reconfigurable. For example, for emulsion droplets functionalized with DNA linkers, the large linker patch that is formed between particles can slow down the motion when the supporting fluid is inhomogeneous ${ }^{61}$ and colloidal joints lose their flexibility with an increasing concentration of dsDNA linkers in the bond area. ${ }^{16}$

To measure the flexibility of larger structures, we assembled CSLBs with complementary dsDNA linkers and imaged chains of three CSLBs, so called trimers, over time. We extracted the position of the individual particles and the opening angle $\theta$ (see inset Fig. 7A) for all frames and calculated the mean squared angular displacement (MSAD) to characterize the flexibility. ${ }^{16}$ Because of the high density of the silica spheres (particle type 1 , Table 1), the particles sedimented to the glass substrate and were therefore confined to move in a quasi-2D plane. To investigate the influence of the membrane homogeneity on the structural flexibility, we compared trimers assembled from CSLBs with homogeneous, fluid bilayer to trimers with bilayers that had spontaneously formed membrane tubes. In the following, we will only show the free energy landscape for $\theta$ from $60^{\circ}$ to $180^{\circ}$ due to the symmetry of a trimer.

For trimers made from CSLBs with smooth lipid bilayers, we found that the particles $(D=2 \mu \mathrm{m})$ move with respect to each other over the full angular range. We analyzed the opening angle $\theta$ for 53 different clusters by tracking the individual particles and calculating $\theta$ for all frames (see inset Fig. 7A). The average value of the flexibility of the trimers is $J=(184 \pm 101) \mathrm{deg}^{2} \mathrm{~s}^{-1}$ (or $\left.(0.03 \pm 0.02) \mu \mathrm{m}^{2} \mathrm{~s}^{-1}\right)$ 

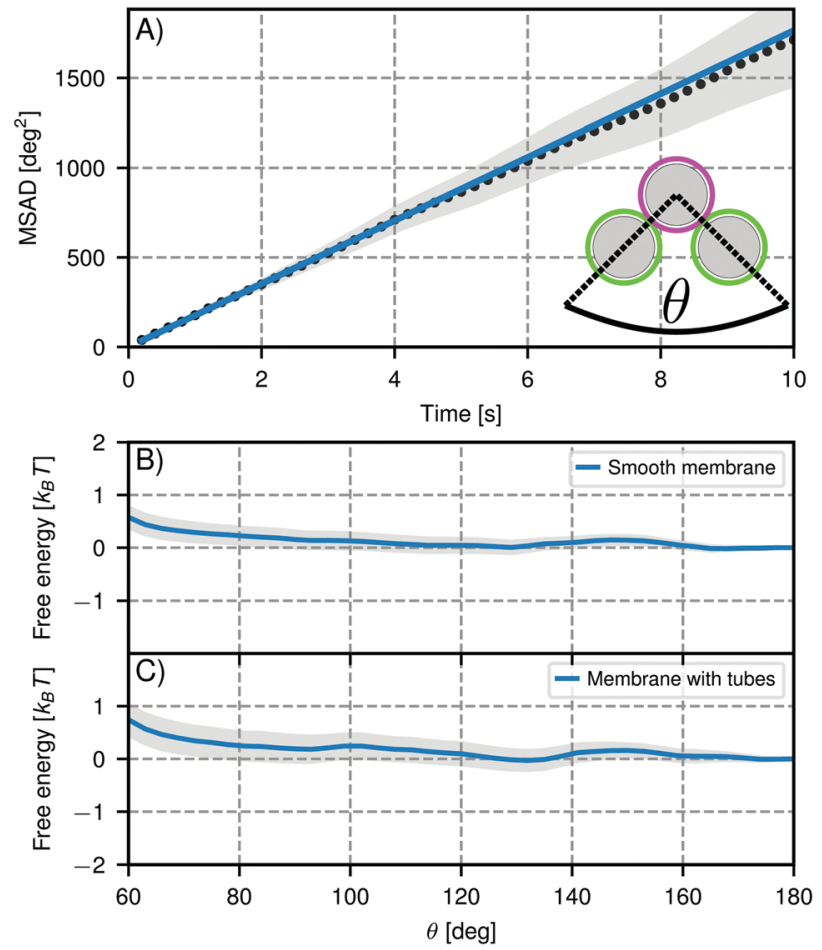

Fig. 7 Mobility of self-assembled trimers. (A) Mean squared angular displacement (MSAD) of the opening angle $\theta$ for a mobile trimer assembled from particles of type 1, Table 1 . The MSAD is linear and we find a joint flexibility $J=(176 \pm 12) \operatorname{deg}^{2} s^{-1}\left(J=(184 \pm 101) \operatorname{deg}^{2} s^{-1}\right.$ when averaging over all experiments). ( $B$ and $C$ ) Free energy as function of opening angle $\theta$ for particles with (B) smooth bilayers and (C) bilayers that have membrane tubes. The grey shaded area marks one standard deviation confidence interval. We analyzed 53 clusters with smooth bilayers and 18 clusters with membrane tubes. In both cases, we find no preference for specific opening angles within the experimental error, meaning the particles are freelyjointed. Note that the slight repulsion at small angles is caused by boundary effects inherent to our analysis method.

and agrees well with previous experiments. ${ }^{16}$ The spread in the flexibility that we observe is likely caused by the spread in dsDNA linker density on the particles. We then determined the free energy using the maximum likelihood estimation of angular displacements method (see Experimental section, as shown in Fig. 7B). We found no preference in the opening angle that is significant with respect to the thermal energy $k_{\mathrm{B}} T$, indicating that the surface is smooth enough to allow the particles to move over one another without restrictions. We observed a boundary effect inherent to the analysis for angles smaller than $60^{\circ}+\sqrt{2 J \tau} \approx 69^{\circ}$ (where $J$ is the joint flexibility) leading to a slight overestimation of the free energy for those angles. In earlier experiments, we found a small preference $\left(0.9 k_{\mathrm{B}} T\right.$ free energy difference) for opening angles around $140^{\circ} .{ }^{16}$ However, at that time we could not accurately determine the confidence interval of the calculated free energy. When re-analyzing the data from ref. 16 using our maximum likelihood method, we noted that the observed free energy difference is within the experimental error (see Fig. S3 in the ESI $\dagger$ ). This is in line with the free energy reported here, which we calculated from a more extensive dataset, and with recent experiments on flexibly linked emulsion droplets, which are freely-jointed like the CSLBs we present here. ${ }^{18}$

To test the robustness of this structural flexibility in the presence of membrane inhomogeneities, we prepared particles with a large number of membrane tubes by washing with inert dsDNA. As shown in Fig. 7(C), this did not alter the flexibility of the clusters: they still explore the full angular range and do not show a significant preferred opening angle, similar to the particles coated with a smooth membrane. Surprisingly, this means that particles stabilized by dsDNA can in principle be used for self-assembly studies despite the fact that the high concentration of dsDNA causes membrane tubes to form, because the tubes do not significantly alter the relative motion of clustered particles.

\section{Summary and conclusions}

We investigated various factors in the preparation protocol of colloid supported lipid bilayers (CSLBs) in view of their emerging use in self-assembly and model membrane studies. Specifically, we focused on realizing a homogeneous and fluid bilayer, while achieving colloidal stability and functionalization with DNA linkers at the same time.

Similar to what has been reported for flat supported lipid bilayers, we found that the quality of the lipid bilayer on colloidal particles critically depends on the material of the particle's surface. The bilayer was not fluid on particles made from polystyrene (with or without carboxyl groups), hematite and TPM particles (with or without carboxyl or amino groups). Colloids featuring a silica surface, on the other hand, were able to host a fluid and homogenous bilayer, at least in the absence of any polymer residues from the synthesis. We furthermore observed that the variation in the substrate curvature does not affect the bilayer formation if it is sufficiently gentle, while excessive surface roughness can hinder the spreading and fusion of SUVs.

Use of PEGylated lipids in the bilayer increased the colloidal stability, but affected the bilayer homogeneity and mobility negatively. Addition of the amphiphilic surfactant SDS led to a disintegration of the bilayer. A better way to provide colloidal stability is by steric stabilisation by excluded volume effects through the insertion of double-stranded inert DNA. Increasing the concentration of dsDNA leads to an increase in colloidal stability.

Finally, we demonstrated that these CSLBs can be functionalized with surface-mobile DNA linkers and assembled them into flexible structures of freely-jointed particles. We found that local bilayer inhomogeneities in the form of membrane tubes do not affect the free energy landscape of the connected particles.

CSLBs with fluid, homogeneous membranes and surfacemobile binding groups have great promise in a wide range of applications and fundamental studies. The fact that the bonded particles can flexibly move with respect to each other opens the door to overcoming equilibration issues previously 
encountered in hit-and-stick processes and assembling structures with internal deformation modes. This enables studying the impact of structural flexibility on the phase behaviour, such as the formation of crystals with new lattices or properties, ${ }^{19-22}$ and the experimental realization of information elements for wet computing. ${ }^{25}$ CSLBs with increased membrane fluidity also mimic biological membranes more closely which may be advantageous for model membrane and cell biology studies ${ }^{8-12}$ smart drug delivery $^{2-4}$ and bio-sensing applications. ${ }^{5,6}$

\section{Author contributions}

MR and RWV contributed equally to the work. MR and RWV performed the experiments and the data analysis. IC performed exploratory experiments. MR, RWV and DJK conceived the experiments and wrote the paper.

\section{Conflicts of interest}

There are no conflicts to declare.

\section{Acknowledgements}

We thank Rachel Doherty and Vera Meester for help with the colloidal syntheses and electron microscopy imaging, and Piermarco Fonda and Luca Giomi for useful discussions. This work was supported by the Netherlands Organization for Scientific Research (NWO/OCW), as part of the Frontiers of Nanoscience program and VENI grant 680-47-431. This project has received funding from the European Research Council (ERC) under the European Union's Horizon 2020 research and innovation program (grant agreement no. 758383).

\section{References}

1 A. L. Troutier and C. Ladavière, An overview of lipid membrane supported by colloidal particles, Adv. Colloid Interface Sci., 2007, 133(1), 1-21.

2 A. M. Carmona-Ribeiro, Preparation and characterization of biomimetic nanoparticles for drug delivery, Nanoparticles in Biology and Medicine: Methods and Protocols, Humana Press, Totowa, NJ, 2012, pp. 283-294.

$3 \mathrm{~J}$. Li, et al., A review on phospholipids and their main applications in drug delivery systems, Asian J. Pharm. Sci., 2015, 10(2), 81-98.

4 S. Savarala, S. Ahmed, M. A. Ilies and S. L. Wunder, Formation and Colloidal Stability of DMPC Supported Lipid Bilayers on $\mathrm{SiO}_{2}$ Nanobeads, Langmuir, 2010, 26(14), 12081-12088.

5 E. T. Castellana and P. S. Cremer, Solid supported lipid bilayers: From biophysical studies to sensor design, Surf. Sci. Rep., 2006, 61(10), 429-444.

6 S. Chemburu, K. Fenton, G. P. Lopez and R. Zeineldin, Biomimetic Silica Microspheres in Biosensing, Molecules, 2010, 15(12), 1932-1957.
7 I. Brouwer, et al., Direct quantitative detection of Doc2binduced hemifusion in optically trapped membranes, Nat. Commun., 2015, 6, 8387.

8 E. Sackmann, Supported membranes: scientific and practical applications, Science, 2007, 271(5245), 43-48.

9 C. Madwar, G. Gopalakrishnan and R. B. Lennox, Interfacing living cells and spherically supported bilayer lipid membranes, Langmuir, 2015, 31(16), 4704-4712.

10 S. Mashaghi, T. Jadidi, G. Koenderink and A. Mashaghi, Lipid Nanotechnology, Int. J. Mol. Sci., 2013, 14, 4242-4282.

11 M. Rinaldin, P. Fonda, L. Giomi and D. J. Kraft, Geometric pinning and antimixing in scaffolded lipid vesicles, 2018, arXiv e-prints.

12 P. Fonda, M. Rinaldin, D. J. Kraft and L. Giomi, Interface geometry of binary mixtures on curved substrates, Phys. Rev. $E, 2018,98,032801$.

13 S. A. Van Der Meulen and M. E. Leunissen, Solid colloids with surface-mobile DNA linkers, J. Am. Chem. Soc., 2013, 135(40), 15129-15134.

14 S. A. J. Van Der Meulen, G. V. Dubacheva, M. Dogterom, R. P. Richter and M. E. Leunissen, Quartz crystal microbalance with dissipation monitoring and spectroscopic ellipsometry measurements of the phospholipid bilayer anchoring stability and kinetics of hydrophobically modified DNA oligonucleotides, Langmuir, 2014, 30(22), 6525-6533.

15 S. A. J. Van der Meulen, G. Helms and M. Dogterom, Solid colloids with surface-mobile linkers, J. Phys.: Condens. Matter, 2015, $27(23), 233101$.

16 I. Chakraborty, V. Meester, C. Van der Wel and D. J. Kraft, Colloidal joints with designed motion range and tunable joint flexibility, Nanoscale, 2017, 9, 7814-7821.

17 L. Feng, L.-L. Pontani, R. Dreyfus, P. Chaikin and J. Brujic, Specificity, flexibility and valence of DNA bonds guide emulsion architecture, Soft Matter, 2013, 9(41), 9816-9823.

18 A. McMullen, M. Holmes-Cerfon, F. Sciortino, A. Y. Grosberg and J. Brujic, Freely jointed polymers made of droplets, Phys. Rev. Lett., 2018, 121, 138002.

19 K. L. Kohlstedt and S. C. Glotzer, Self-assembly and tunable mechanics of reconfigurable colloidal crystals, Phys. Rev. E Statistical, Nonlinear, and Soft Matter Physics, 2013, 87(3), 032305.

20 D. Ortiz, K. L. Kohlstedt, T. D. Nguyen and S. C. Glotzer, Self-assembly of reconfigurable colloidal molecules, Soft Matter, 2014, 10(20), 3541-3552.

21 F. Smallenburg, L. Filion and F. Sciortino, Erasing no-man's land by thermodynamically stabilizing the liquid-liquid transition in tetrahedral particles, Nat. Phys., 2014, 10(9), 653-657.

22 H. Hu, P. S. Ruiz and R. Ni, Entropy Stabilizes Floppy Crystals of Mobile DNA-Coated Colloids, Phys. Rev. Lett., 2018, $120(4), 048003$.

23 J. D. Joannopoulos, P. R. Villeneuve and S. Fan, Photonic crystals: putting a new twist on light, Nature, 1997, 386(6621), 143-149.

24 Y. Lin, et al., Photonic band structure of colloidal crystal self-assembled in hollow core optical fiber, Appl. Phys. Lett., 2005, 86(12), 121106. 
25 C. L. Phillips, et al., Digital colloids: reconfigurable clusters as high information density elements, Soft Matter, 2014, 10(38), 7468-7479.

26 M. Hadorn and P. Eggenberger Hotz, DNA-Mediated SelfAssembly of Artificial Vesicles, PLoS One, 2010, 5(3), e9886.

27 P. A. Beales, J. Nam and T. K. Vanderlick, Specific adhesion between dna-functionalized janus vesicles: size-limited clusters, Soft Matter, 2011, 7, 1747-1755.

28 L. Parolini, et al., Volume and porosity thermal regulation in lipid mesophases by coupling mobile ligands to soft membranes, Nat. Commun., 2015, 6, 5948.

29 V. T. Milam, Oligonucleotide-based recognition in colloidal systems - opportunities and challenges, Curr. Opin. Colloid \& In. Sci., 2016, 26, 75-83.

30 Y. Wang, et al., Colloids with valence and specific directional bonding, Nature, 2012, 491(7422), 51-55.

31 W. B. Rogers and V. N. Manoharan, Programming colloidal phase transitions with DNA strand displacement, Science, 2015, 347(6222), 639-642.

32 Y. Wang, et al., Crystallization of DNA-coated colloids, Nat. Commun., 2015, 6, 7253.

33 N. B. Schade, et al., Tetrahedral Colloidal Clusters from Random Parking of Bidisperse Spheres, Phys. Rev. Lett., 2013, 110(14), 148303.

34 Y. Zhang, et al., Sequential self-assembly of DNA functionalized droplets, Nat. Commun., 2017, 8(1), 21.

35 T. Sugimoto and K. Sakata, Preparation of monodisperse pseudocubic $\alpha-\mathrm{Fe}_{2} \mathrm{O}_{3}$ particles from condensed ferric hydroxide gel, J. Colloid Interface Sci., 1992, 152(2), 587-590.

36 L. Rossi, et al., Cubic crystals from cubic colloids, Soft Matter, 2011, 7(9), 4139-4142.

37 V. Meester and D. J. Kraft, Spherical, Dimpled, and Crumpled Hybrid Colloids with Tunable Surface Morphology, Langmuir, 2016, 32(41), 10668-10677.

38 C. Van der Wel, et al., Preparation of colloidal organosilica spheres through spontaneous emulsification, Langmuir, 2017, 33(33), 8174-8180.

39 R. P. Doherty and D. J. Kraft, One-pot surfactant-free synthesis of organosilica colloids with various surface functional groups, in preparation.

40 J. Appel, S. Akerboom, R. G. Fokkink and J. Sprakel, Facile One-Step Synthesis of Monodisperse Micron-Sized Latex Particles with Highly Carboxylated Surfaces, Macromol. Rapid Commun., 2013, 34(16), 1284-1288.

41 P. S. Cremer and S. G. Boxer, Formation and Spreading of Lipid Bilayers on Planar Glass Supports, J. Phys. Chem. B, 1999, 103(13), 2554-2559.

42 A. Y. Antipina and A. A. Gurtovenko, Molecular-level insight into the interactions of DNA with phospholipid bilayers: barriers and triggers, RSC Adv., 2016, 6, 36425-36432.

43 C. Van der Wel, et al., Lipid membrane-mediated attraction between curvature inducing objects, Sci. Rep., 2016, 6, 32825.

44 D. Axelrod, D. Koppel, J. Schlessinger, E. Elson and W. Webb, Mobility measurement by analysis of fluorescence photobleaching recovery kinetics, Biophys. J., 1976, 16(9), 1055-1069.
45 D. B. Allan, T. Caswell, N. C. Keim and C. M. Van der Wel, trackpy: Trackpy v0.4.1, 2018, URL https://doi.org/10.5281/ zenodo.1226458.

46 C. M. Van der Wel, Lipid Mediated Colloidal Interactions, Casimir PhD Series, Leiden University, The Netherlands, 2017.

47 R. Sarfati, J. Bławzdziewicz and E. R. Dufresne, Maximum likelihood estimations of force and mobility from single short Brownian trajectories, Soft Matter, 2017, 13(11), 2174-2180.

48 D. Foreman-Mackey, D. W. Hogg, D. Lang and J. Goodman, emcee: The MCMC Hammer, 2013, 125, 306, ArXiv e-prints.

49 G. Gopalakrishnan, I. Rouiller, D. R. Colman and R. B. Lennox, Supported bilayers formed from different phospholipids on spherical silica substrates, Langmuir, 2009, 25(10), 5455-5458.

50 R. P. Richter, R. Bérat and A. R. Brisson, Formation of solidsupported lipid bilayers: an integrated view, Langmuir, 2006, 22(8), 3497-3505.

51 E. Sackmann, Supported Membranes: Scientific and Practical Applications, Science, 1996, 271(5245), 43-48.

52 J. Rädler, H. Strey and E. Sackmann, Phenomenology and Kinetics of Lipid Bilayer Spreading on Hydrophilic Surfaces, Langmuir, 1995, 11(11), 4539-4548.

53 I. Gözen and A. Jesorka, Instrumental methods to characterize molecular phospholipid films on solid supports, Anal. Chem., 2012, 84(2), 822-838.

54 R. Macháň and M. Hof, Lipid diffusion in planar membranes investigated by fluorescence correlation spectroscopy, Biochim. Biophys. Acta, 2010, 1798(7), 1377-1391.

55 Y. Jing, H. Trefna, M. Persson, B. Kasemo and S. Svedhem, Formation of supported lipid bilayers on silica: relation to lipid phase transition temperature and liposome size, Soft Matter, 2014, 10(1), 187-195.

56 S. I. R. Castillo, et al., Silica cubes with tunable coating thickness and porosity: From hematite filled silica boxes to hollow silica bubbles, Microporous Mesoporous Mater., 2014, 195, 75-86.

57 T. Cha, A. Guo and X.-Y. Zhu, Formation of supported phospholipid bilayers on molecular surfaces: Role of surface charge density and electrostatic interaction, Biophys. J., 2006, 90(4), 1270-1274.

58 P. G. De Gennes, Polymers at an interface; a simplified view, Adv. Colloid Interface Sci., 1987, 27(3-4), 189-209.

59 S. Upadhyayula, et al., Coatings of polyethylene glycol for suppressing adhesion between solid microspheres and flat surfaces, Langmuir, 2012, 28(11), 5059-5069.

60 C. Van der Wel, et al., Surfactant-free colloidal particles with specific binding affinity, Langmuir, 2017, 33(38), 9803-9810.

61 C. Van Der Wel, G. L. Van De Stolpe, R. W. Verweij and D. J. Kraft, Micrometer-sized TPM emulsion droplets with surface-mobile binding groups, J. Phys.: Condensed Matter, 2018, 30(9), 094005.

62 C. A. Naumann, et al., The polymer-supported phospholipid bilayer: Tethering as a new approach to substrate- membrane stabilization, Biomacromolecules, 2002, 3(1), 27-35.

63 M. Tanaka and E. Sackmann, Polymer-supported membranes as models of the cell surface, Nature, 2005, 437(7059), 656-663.

64 M. Tanaka, Polymer-Supported Membranes: Physical Models of Cell Surfaces, MRS Bull., 2006, 31, 513-520. 
65 M. L. Wagner and L. K. Tamm, Tethered Polymer-Supported Planar Lipid Bilayers for Reconstitution of Integral Membrane Proteins: Silane-Polyethyleneglycol-Lipid as a Cushion and Covalent Linker, Biophys. J., 2000, 79(3), 1400-1414.

66 M. A. Deverall, et al., Transbilayer coupling of obstructed lipid diffusion in polymer-tethered phospholipid bilayers, Soft Matter, 2008, 4, 1899-1908.

67 R. Lipowsky, Bending of membranes by anchored polymers, Europhys. Lett., 1995, 30(4), 197.

68 N. Geerts and E. Eiser, DNA-functionalized colloids: physical properties and applications, Soft Matter, 2010, 6(19), 4647-4660.

69 M.-P. Valignat, O. Theodoly, J. C. Crocker, W. B. Russel and P. M. Chaikin, Reversible self-assembly and directed assembly of DNA-linked micrometer-sized colloids, Proc. Natl. Acad. Sci. U. S. A., 2005, 102(12), 4225-4229.

70 P. L. Biancaniello, J. C. Crocker, D. A. Hammer and V. T. Milam, DNA-mediated phase behavior of microsphere suspensions, Langmuir, 2007, 23(5), 2688-2693.

71 G. Nordlund, R. Lönneborg and P. Brzezinski, Formation of Supported Lipid Bilayers on Silica Particles Studied Using Flow Cytometry, Langmuir, 2009, 25(8), 4601-4606.
72 A. Tan, A. Ziegler, B. Steinbauer and J. Seelig, Thermodynamics of sodium dodecyl sulfate partitioning into lipid membranes, Biophys. J., 2002, 83(3), 1547-1556.

73 T. Drobek, N. D. Spencer and M. Heuberger, Compressing PEG brushes, Macromolecules, 2005, 38(12), 5254-5259.

74 F. Meng, G. H. M. Engbers and J. Feijen, Polyethylene glycolgrafted polystyrene particles, J. Biomed. Mater. Res., Part A, 2004, 70(1), 49-58.

75 O. Garbuzenko, Y. Barenholz and A. Priev, Effect of grafted PEG on liposome size and on compressibility and packing of lipid bilayer, Chem. Phys. Lipids, 2005, 135(2), 117-129.

76 S. Angioletti-Uberti, P. Varilly, B. M. Mognetti and D. Frenkel, Mobile linkers on DNA-coated colloids: Valency without patches, Phys. Rev. Lett., 2014, 113(12), 128303.

77 R. Lipowsky, Spontaneous tubulation of membranes and vesicles reveals membrane tension generated by spontaneous curvature, Faraday Discuss., 2013, 161, 305-331.

78 S. I. Nakano, M. Fujimoto, H. Hara and N. Sugimoto, Nucleic acid duplex stability: Influence of base composition on cation effects, Nucleic Acids Res., 1999, 27(14), 2957-2965. 